\title{
Stabilization of negative capacitance in ferroelectric capacitors with and without a metal interlayer
}

\author{
T. Rollo ${ }^{1}$, F. Blanchini ${ }^{2}$, G. Giordano ${ }^{3}$, R. Specogna ${ }^{1}$, D. Esseni ${ }^{1}$ \\ ${ }^{1}$ DPIA, University of Udine, Via delle Scienze 206, 33100 Udine, Italy; email: david.esseni @ uniud.it \\ ${ }^{2}$ DMIF, University of Udine, Via delle Scienze 206, 33100 Udine, Italy \\ ${ }^{3}$ DII, University of Trento, via Sommarive 9, 38123 Povo (TN), Italy
}

\begin{abstract}
The negative capacitance operation of a ferroelectric material is not only an intriguing material science topic, but also a property with important technological applications in nanoscale electron devices. Despite the growing interest for possible applications, the very existence of negative capacitance is still actively debated, even because experimental results for ferroelectric capacitors with or without a metal interlayer led to quite contradicting indications. Here we present a comprehensive analysis of the $\mathrm{NC}$ operation in ferroelectric capacitors and provide new insights about the discrepancies observed in experiments. Our models duly account for the three-dimensional nature of the problem and show a good agreement with several aspects of recent experiments. Our results also demonstrate that traps at the ferroelectric-dielectric interface play an important role in the feasibility of a stable negative capacitance operation in ferroelectric capacitors.
\end{abstract}

\section{Introduction}

The basic idea behind the use of ferroelectric materials in nanoscale transistors stems from the fact that, thanks to the negative capacitance (NC) operation, the voltage swing necessary to operate the transistors can be reduced, ${ }^{1-3}$ thus enabling improved energy efficiency for CMOS circuits. ${ }^{4,5}$ An industrial level demonstration of the NC operation in CMOS transistors was recently reported for a 14-nm FinFET technology, ${ }^{6}$ with an analysis of the device and circuit level advantages further discussed in. ${ }^{7}$ Moreover, several papers have started addressing diverse design aspects related to NC field effect transistors. ${ }^{8-10}$

Despite some encouraging experimental results, however, the stable NC operation of the ferroelectric is still quite controversial. ${ }^{11}$ In fact, recent studies in Metal-Ferroelectric-Insulator-Metal (MFIM) capacitors reported a hysteresis free, direct measurement of the negative capacitance branch of a thin ferroelectric layer. ${ }^{12,13}$ However, in similarly recent publications focused on Metal-Ferroelectric-Metal-Insulator-Metal (MFMIM) systems or on ferroelectric capacitors externally connected to a MOSFET authors either negated any evidence of the NC operation, ${ }^{14}$ or affirmed that the measured steep slope transistor operation was due to domain switching and, as such, invariably accompanied by hysteresis. ${ }^{15-17}$

The discrepancy between experiments in MFIM and MFMIM systems is not entirely unexpected, in fact a recent theoretical investigation suggests that MFMIM capacitors are inherently more prone than MFIM systems to domain nucleation. ${ }^{18}$ The analysis in, ${ }^{18}$ however, was restricted to a one-dimensional, rigidly periodic system and, moreover, conclusions were drawn by inspecting the free energy landscapes, instead of examining the actual ferroelectric dynamic equations of the MFIM and MFMIM systems.

In this paper we present a comprehensive analysis of the dynamics and possible stabilisation of a ferroelectric layer inserted either in a MFIM or in a MFMIM structure, which is a broadly extended version of the concise contribution reported in. ${ }^{19}$ To this purpose we have developed a model for the depolarisation energy that fully accounts for the three-dimensional nature of the electrostatics in a realistic device. Then we use the multi-domain Landau-Ginzburg-Devonshire theory (LGD) and derive analytical or quasi-analytical conditions for a stable NC operation, that explain the different behavior of a MFIM compared to a MFMIM capacitor. Our models are validated by a good agreement with several aspects of recent experiments. ${ }^{12,13}$ Finally we investigate the influence of possible traps at the ferroelectric-dielectric interface, and argue that traps not only help explain some experimental features, but also discriminate between a quasi-static and a dynamic NC operation. 
a

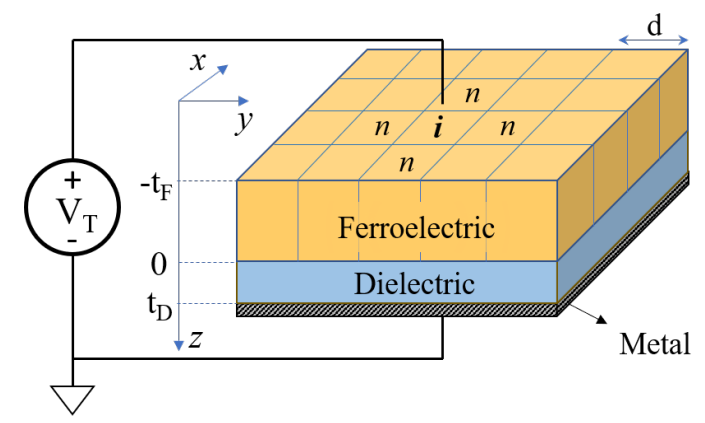

c

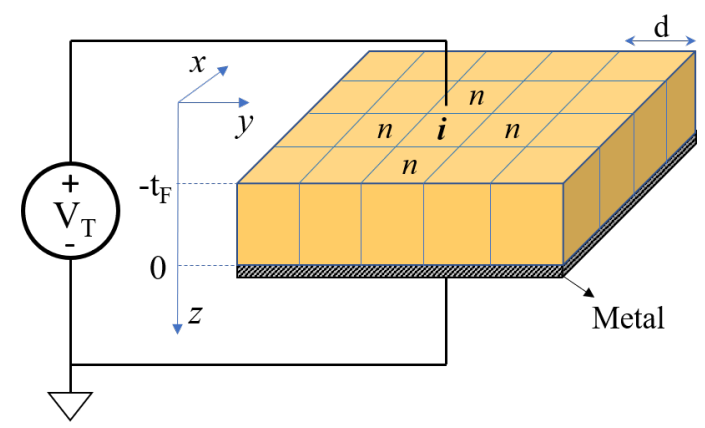

b

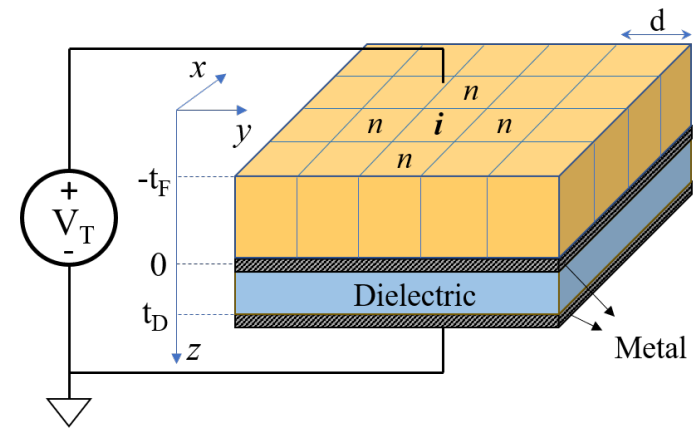

d

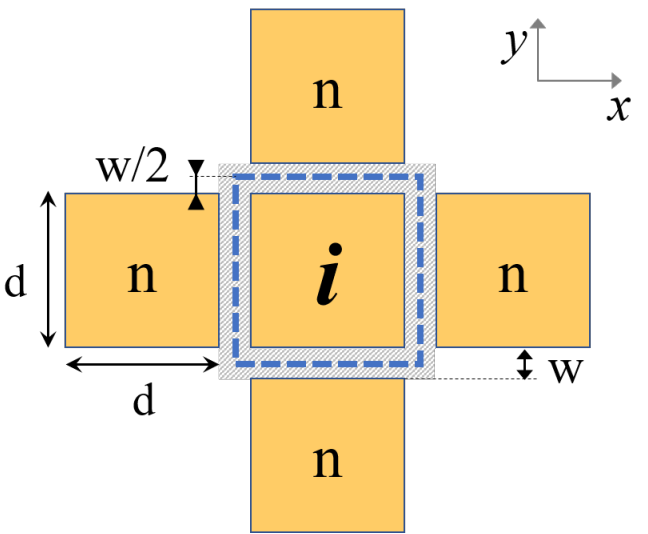

Figure 1: Ferroelectric capacitors and related symbols. a. Metal-Ferroelectric-Insulator-Metal (MFIM) and reference coordinate system. b. Metal-Ferroelectric-Metal-Insulator-Metal (MFIMIM) system. c. Metal-Ferroelectric-Metal (MFM) structure. The top metal contact is not shown for clarity. $t_{F}$ and $t_{D}$ denote respectively the ferroelectric and dielectric thickness, $d$ is the domain side of a square domain of area $d^{2}$, and $V_{T}$ is the externally applied voltage. $V_{D}(\bar{r})$ is the electrostatic potential at the oxide interface (i.e. at $z=0$ ), that depends on $\bar{r}=(x, y)$ in a MFIM system, whereas it is independent of $\bar{r}$ in a MFMIM capacitor. d. Sketch of the ferroelectric domain $i$ and its nearest neighbors domains $n$ in the $x-y$ plane. The shaded area illustrates the domain-wall region, where $w$ denotes the width, and the dashed blue line delimits the region used to compute the domain wall energy $u_{W, i}$ in Eq.4.

\section{Free energy and dynamic equations}

In the analysis of the ferroelectric capacitors sketched in Fig.1 we assume that the spontaneous polarisation $P$ lies along the $z$ direction, and we write the free energy per unit volume of the ferroelectric by following ${ }^{18}$

$$
u_{F}=\alpha P^{2}+\beta P^{4}+\gamma P^{6}+k|\operatorname{grad} P|^{2}+\frac{\varepsilon_{0} \varepsilon_{F}}{2} E_{F}^{2}
$$

where $\alpha, \beta$ and $\gamma$ are the ferroelectric anisotropy constants, $\varepsilon_{0}$ is the vacuum permittivity, $E_{F}, \varepsilon_{F}$ are respectively the electric field and relative background permittivity of the ferroelectric, while $k$ is the coupling constant governing the domain wall energy and $\operatorname{grad} P$ denotes the gradient of $P$. The total polarisation in the ferroelectric is thus given by $P_{T}=P+\left(\varepsilon_{F}-1\right) \varepsilon_{0} E_{F}$ and the electric displacement is $D=P+\varepsilon_{F} \varepsilon_{0} E_{F} .{ }^{20}$ We will assume that the ferroelectric has a second-order phase transition with $\alpha<0, \beta>0$. When we consider the ferroelectric capacitors of Fig. 1 the overall electrostatic energy consists of the three contributions ${ }^{21}$

$$
\mathscr{U}_{F}=\frac{V_{T}}{2} \int_{A} \varepsilon_{0} \varepsilon_{F} E_{F, T}(\bar{r}) d \bar{r}, \quad \mathscr{U}_{B}=-V_{T}\left[d^{2} \sum_{j=1}^{n_{D}} P_{j}+\int_{A} \varepsilon_{0} \varepsilon_{F} E_{F, T}(\bar{r}) d \bar{r}\right], \quad \mathscr{U}_{D}=\sum_{j=1}^{n_{D}} \int_{D_{j}} \frac{P_{j} V_{D}(\bar{r})}{2} d \bar{r}[J]
$$

namely the ferroelectric self-energy, $\mathscr{U}_{F}$, the $\mathscr{U}_{B}$ related to the external battery, and the electrostatic energy, $\mathscr{U}_{D}$, due to the dielectric region, which is zero in a MFM structure. Denoting by $t_{F}$ the ferroelectric thickness, $E_{F, T}(\bar{r})=E_{F, z}\left(\bar{r},-t_{F}\right)$ in Eq.2 is the electric field at the top metal interface and $n_{D}$ is the number of domains. When we sum $\mathscr{U}_{F}, \mathscr{U}_{B}, \mathscr{U}_{D}$ and normalise to the domain area $d^{2}$ we obtain

$$
U_{E T}=-\frac{V_{T}}{2} \frac{1}{d^{2}} \int_{A} \varepsilon_{0} \varepsilon_{F} E_{F, T}(\bar{r}) d \bar{r}-V_{T} \sum_{j=1}^{n_{D}} P_{j}+\frac{1}{d^{2}} \sum_{j=1}^{n_{D}} \int_{D_{j}} \frac{P_{j} V_{D}(\bar{r})}{2} d \bar{r}\left[J / m^{2}\right]
$$


As for the domain wall energy, the polarisation is assumed to be essentially constant within each domain, so that $\operatorname{grad} P$ in Eq.1 is non null only in the domain wall region of Fig.1(d). The contribution $u_{W, i}$ to the domain wall energy can thus be written as

$$
u_{W, i}=\sum_{n} k\left(\frac{P_{i}-P_{n}}{w}\right)^{2}
$$

where $w$ is the domain wall width shown in Fig.1(d), which we assume to be small enough to justify the discretized form of $\operatorname{grad} P$ in Eq.4 and, in particular, much smaller than $d$. We can now integrate $u_{W, i}$ over the domain wall region inside the blue line in Fig.1(d) and along $t_{F}$, and then normalise to the domain area $d^{2}$, so as to obtain the domain wall energy per unit area

$$
U_{W}=\sum_{j=1}^{n_{D}}\left[\frac{t_{F}}{2 d} \sum_{n} \frac{k}{w}\left(P_{j}-P_{n}\right)^{2}\right]\left[J / m^{2}\right]
$$

The difference between the MFM, MFIM and MFMIM systems is in the $U_{E T}$ defined in Eq.3. In the MFM case the last term in Eq.3 is zero and $E_{F, T}=V_{T} / t_{F}$, so that $U_{E T}=-V_{T} \sum_{j=1}^{n_{D}} P_{j}-\left(n_{D} C_{F} V_{T}^{2}\right) / 2$ with $C_{F}=\varepsilon_{0} \varepsilon_{F} / t_{F}$. For the MFMIM structure the metal interlayer results in a one-dimensional electrostatics, so that $E_{F, T}$ and $V_{D}$ are independent of $\bar{r}$ and given by $E_{F, T}=\left(C_{D} V_{T}-P_{A V}\right) /\left(t_{F} C_{0}\right), V_{D}=\left(C_{F} V_{T}+P_{A V}\right) / C_{0},{ }^{18}$ where $P_{A V}=\left(\sum_{j=1}^{n_{D}} P_{j}\right) / n_{D}$ is the average polarisation, $C_{D}=\varepsilon_{0} \varepsilon_{D} / t_{D}$ (where $t_{D}$ denotes the dielectric thickness) and $C_{0}=\left(C_{D}+C_{F}\right)$. For the MFIM system, instead, the calculation of the ferroelectic and dielectric field is a three-dimensional problem that demands a numerical evaluation. We show in Supplementary Section S1 that for both the MFMIM and the MFIM system the electrostatic energy reads

$$
U_{E T}=U_{d e p}-V_{T} \frac{C_{D}}{C_{0}} \sum_{j}^{n_{D}} P_{j}-\frac{C_{S} V_{T}^{2}}{2} n_{D}
$$

where $C_{S}=\left(C_{F} C_{D}\right) /\left(C_{F}+C_{D}\right)$. Here $U_{d e p}$ denotes the depolarisation energy defined as

$$
\text { MFMIM: } U_{d e p}=\frac{n_{D} P_{A V}^{2}}{2 C_{0}} \quad \text { MFIM: } U_{d e p}=\frac{1}{2} \sum_{j, h=1}^{n_{D}} \frac{P_{j} P_{h}}{C_{j, h}}
$$

where the capacitances $C_{j, h}$ are defined in Eq.S4 of Supplementary Section S1, they obey the sum rules in Eq.S7, and all $1 / C_{j, h}$ tend to zero when $t_{D}$ tends to zero. As it can be seen, the depolarisation energy $U_{d e p}$ vanishes when the dielectric thickness $t_{D}$ tends to zero.

For all the systems in Fig.1 the overall free energy is $U_{T}=\sum_{j=1}^{n_{D}}\left(\alpha P_{j}^{2}+\beta P_{j}^{4}+\gamma P_{j}^{6}\right)+U_{W}+U_{E T}$ and the corresponding dynamic equations read

$$
\begin{aligned}
& \text { MFM: } t_{F} \rho \frac{d P_{i}}{d t}=-\frac{\partial U_{T}}{\partial P_{i}}=\underbrace{-\left(2 \alpha P_{i}+4 \beta P_{i}^{3}+6 \gamma P_{i}^{5}\right) t_{F}-\frac{t_{F}}{d} \sum_{n} \frac{k}{w}\left(P_{i}-P_{n}\right)}_{=\partial U_{L G D}}+V_{T}(t) \\
& \text { MFMIM: } \quad t_{F} \rho \frac{d P_{i}}{d t}=\partial U_{L G D}-\frac{1}{n_{D} C_{0}} \sum_{j=1}^{n_{D}} P_{j}+\frac{C_{D}}{C_{0}} V_{T}(t) \\
& \text { MFIM: } \quad t_{F} \rho \frac{d P_{i}}{d t}=\partial U_{L G D}-\frac{1}{2} \sum_{j=1}^{n_{D}}\left[\frac{1}{C_{i, j}}+\frac{1}{C_{j, i}}\right] P_{j}+\frac{C_{D}}{C_{0}} V_{T}(t)
\end{aligned}
$$

where $\rho$ is the resistivity governing the ferroelectric domain dynamics. It is straightforward to verify that, when the dielectric thickness $t_{D}$ tends to zero, $1 / C_{0}$ and $1 / C_{i, j}$ tend to zero while $\left[C_{D} / C_{0}\right]$ tends to one, so that Eq. $8 \mathrm{~b}$ and Eq.8c simplify to Eq.8a. Moreover for $n_{D}=1$ Eq. $8 \mathrm{~b}$ and Eq.8c are identical, in fact the MFMIM and MFIM systems are equivalent, the domain wall energy is zero and Eq.8b, Eq.8c simplify to the well known single domain equation. ${ }^{18}$ 


\section{Conditions for a stable NC operation}

Throughout this paper we employ a definition of the NC operation consisting in the polarization $P_{i}$ of all domains being zero at zero external voltage $V_{T}$, which ensures a hysteresis-free behavior also in the multi-domain picture. If the ferroelectric is stabilized in a region where $P_{i}$ is not zero for most domains but $\partial^{2} G\left(P_{i}\right) / \partial^{2} P_{i}$ is negative (with $G\left(P_{i}\right)=\left(\alpha_{i} P_{i}^{2}+\beta_{i} P_{i}^{4}+\gamma_{i} P_{i}^{6}\right)$, an NC operation can still be claimed, albeit in the presence of hysteresis. The stable NC operation can be evaluated by inspecting the eigenvalues of the Jacobian matrices ${ }^{1}, \mathbf{J}$, of the dynamic systems in Eqs.8a, 8b and 8c evaluated for $P_{i}=0$ in all domains. Here it should be noticed that analysing the stability of the equilibrium at $P_{i}=0$ and $V_{T}=0$ is not restrictive. In fact, as we show in the Supplementary Section $\mathrm{S} 4$, stability in this case implies stability of the equilibrium for any other constant value of $V_{T}$. The Jacobian matrices read

$$
\begin{gathered}
\mathbf{J}_{M F M}=\frac{1}{\rho t_{F}}\left[-2 \alpha t_{F} \mathbf{I}-\frac{t_{F} k}{d w} \mathbf{L}\right] \\
\mathbf{J}_{M F M I M}=\frac{1}{\rho t_{F}}\left[-2 \alpha t_{F} \mathbf{I}-\frac{t_{F} k}{d w} \mathbf{L}-\frac{\mathbf{O}_{d e p}}{n_{D} C_{0}}\right] \\
\mathbf{J}_{M F I M}=\frac{1}{\rho t_{F}}\left[-2 \alpha t_{F} \mathbf{I}-\frac{t_{F} k}{d w} \mathbf{L}-\mathbf{C}_{d e p}\right]
\end{gathered}
$$

where $\mathbf{I}$ is the $n_{D}$ by $n_{D}$ identity matrix, while $\mathbf{L}$ is the Laplacian matrix ${ }^{2}$. The matrix $\mathbf{O}_{d e p}$ has all entries equal to one, whereas $\mathbf{C}_{d e p}$ is defined as

$$
C_{d e p}(i, j)=\frac{1}{2}\left[\frac{1}{C_{i, j}}+\frac{1}{C_{j, i}}\right]
$$

The matrices $\mathbf{O}_{d e p}$ and $\mathbf{C}_{d e p}$ stem from the depolarisation energy $U_{d e p}$ in Eq.7, and are very different for a MFMIM and a MFIM system. The eigenvalues of the symmetric $\mathbf{J}$ matrices in Eq.9 are real valued and, for a stable $\mathrm{NC}$ operation, it is required that the largest eigenvalue $\sigma_{\max }(\mathbf{J})$ of the Jacobian matrix evaluated for all $P_{i}=0$ be negative. ${ }^{22}$ This results in the equivalent stability conditions

$$
\begin{array}{cc}
\text { MFM: } & \frac{k}{d w} \sigma_{\min }(\mathbf{L})>2|\alpha| \\
\text { MFMIM: } & \sigma_{\min }\left[\frac{t_{F} k}{d w} \mathbf{L}+\frac{\mathbf{O}_{d e p}}{n_{D} C_{0}}\right]>2|\alpha| t_{F} \\
\text { MFIM: } & \sigma_{\min }\left[\frac{t_{F} k}{d w} \mathbf{L}+\mathbf{C}_{d e p}\right]>2|\alpha| t_{F}
\end{array}
$$

where $\sigma_{\min }(\mathbf{M})$ denotes the smallest eigenvalue of the matrix $\mathbf{M}$.

We now recall that the eigenvalues of $\mathbf{L}$ are known analytically in our case (since we are dealing with a rectangular grid) and the smallest and second smallest eigenvalue are $\sigma_{0}(\mathbf{L})=0$ and $\sigma_{1}(\mathbf{L})=\left[2 \sin \left(\pi /\left(2 \sqrt{n_{D}}\right)\right)\right]^{2} .^{23}$ This implies that, as expected, the MFM system is always unstable for all $P_{i}=0$.

For the MFMIM system we show in Supplementary Section S2 that, due to the peculiar form of the matrix $\mathbf{O}_{d e p}$, one can derive the analytical (necessary and sufficient) condition for a stable NC operation given by

$$
\min \left\{\frac{1}{C_{0}}, \frac{t_{F} k}{d w}\left[2 \sin \left(\pi /\left(2 \sqrt{n_{D}}\right)\right)\right]^{2}\right\}>2|\alpha| t_{F}
$$

Eq.12 shows that in the MFMIM system the effect of the depolarisation energy is very limited, in fact $\mathbf{O}_{\text {dep }}$ can only eliminate the influence of $\sigma_{0}(\mathbf{L})=0$ but not the influence of $\sigma_{1}(\mathbf{L})$. Eq.12 also affirms that the condition $\left(1 / C_{0}\right)>2|\alpha| t_{F}$ is necessary for the stability of the MFMIM system. Moreover, for a relatively large number of domains such that $\sin \left(\pi /\left(2 \sqrt{n_{D}}\right) \simeq \pi /\left(2 \sqrt{n_{D}}\right)\right.$, Eq.12 suggests that a stable NC operation for the MFMIM system requires $k / w$ values that increase proportionally to $n_{D}$, hence to the device area.

\footnotetext{
${ }^{1}$ The Jacobian matrix of the system of dynamic equations $d P_{i} / d t=f_{i}\left(P_{1}, \cdots P_{n D}\right)$ is defined component-wise as $J(i, j)=\partial f_{i} / \partial P_{j}$.

${ }^{2} \mathbf{L}$ is defined component-wise as $\mathbf{L}(i, j)=-1$ if domain $j$ is a neighbour of domain $i$ and $\mathbf{L}(i, j)=0$ otherwise (see Fig.1(d)), and $\mathbf{L}(i, i)=-\sum_{j \neq i} \mathbf{L}(i, j)$.
} 
For the MFIM structure it is not possible to derive analytical eigenvalues and stability conditions from Eq.11c, but a numerical analysis shows that $\mathbf{C}_{d e p}$ has a much larger influence on $\mathrm{NC}$ stabilisation than $\mathbf{O}_{d e p}$ has for the MFMIM system. Moreover we show in Supplementary Section S3 that even for the MFIM system the inequality $\left(1 / C_{0}\right)>2|\alpha| t_{F}$ is still a necessary condition for a stable NC operation. It is interesting to notice that this is the stability condition previously derived for a single domain system. ${ }^{18}$

Ferroelectric materials may have domain to domain statistical variations of the ferroelectric anisotropy constants, whose influence on the stable NC operation is addressed in Supplementary Section S5.

a

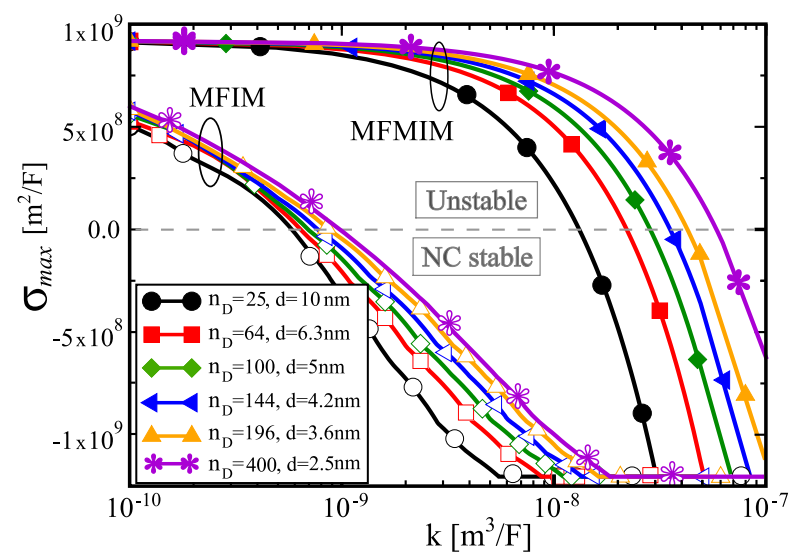

c

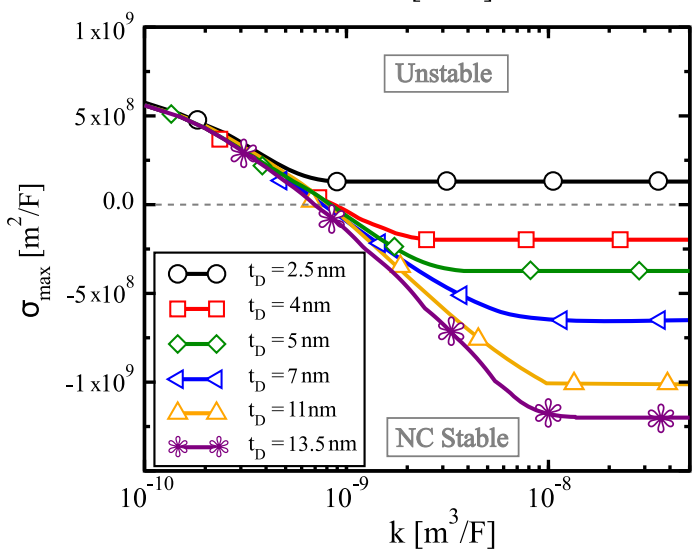

b

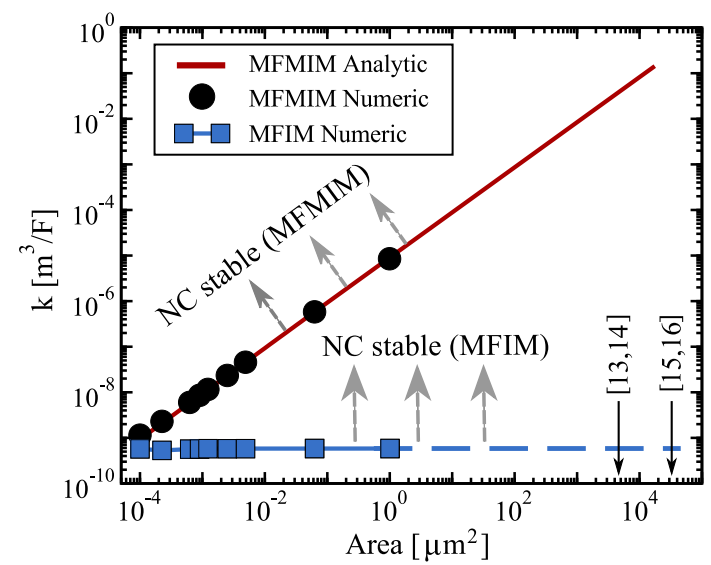

d

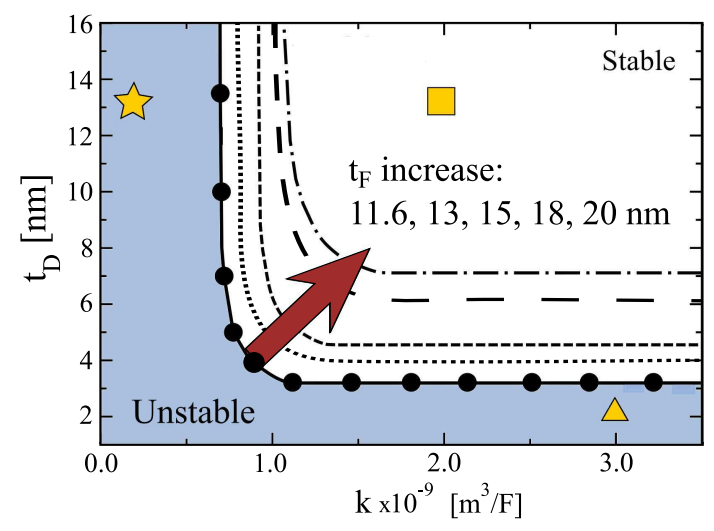

Figure 2: Eigenvalues of the Jacobian matrix and design space for stable NC operation: a. Largest eigenvalue $\sigma_{\max }$ of the Jacobian matrix for all $P_{i}=0$ versus the domain wall coupling factor $k$ for either a MFIM (numerically calculated) or a MFMIM structure. Capacitor area is $\mathrm{A}=2500 \mathrm{~nm}^{2}$ and results are shown for different combinations of $d$ and $n_{D}$. Stable NC operation corresponds to $\sigma_{\max }<0$. b. Minimum coupling factor $k$ necessary for a stable NC operation versus the capacitor area for either a MFMIM or a MFIM structure. For the MFIM structure results have been calculated numerically from the condition $\sigma_{\max }<0$, while for the MFMIM structure results stem from Eq.12. Domain size is $d=5 \mathrm{~nm}$, thus Area $=d^{2} n_{D}$. Please notice the large areas corresponding to recent experiments in. ${ }^{13-16}$ c. Maximum eigenvalue $\sigma$ versus coupling factor $k$ obtained from numerical simulations for a MFIM structure having different $\mathrm{Ta}_{2} \mathrm{O}_{5}$ thicknesses $t_{D}$. Ferroelectric thickness, domain number $n_{D}$ and domain area $d^{2}$ area set to $t_{F}=11.6 \mathrm{~nm}, n_{D}=100$ and $d^{2}=25 \mathrm{~nm}^{2}$. d. Regions for stable NC operation for a MFIM structure in the $t_{D}$ versus $k$ plane and for different $t_{F}$ values. Filled circles correspond to $t_{F}=11.6 \mathrm{~nm}$. For larger $t_{F}$ values the minimum $t_{D}$ required for stability increases, as predicted by the necessary condition $\left(1 / C_{0}\right)>2|\alpha| t_{F}$. Area is $\mathrm{A}=2500 \mathrm{~nm}^{2}$ and $n_{D}=100$. The star, square and triangle symbols identify the $t_{D}$ and $k$ values corresponding to some of the simulations in Fig.3, and are discussed discussed in the text.

\section{Physical insight and design space}

All the simulation results reported in this work were obtained for $\varepsilon_{F}=33, \varepsilon_{D}=23.5, t_{F}=11.6 \mathrm{~nm}, t_{D}=13.5 \mathrm{~nm}$, $\alpha=-4.6 \cdot 10^{8} \mathrm{~m} / \mathrm{F}, \beta=9.8 \cdot 10^{9} \mathrm{~m}^{5} / \mathrm{C}^{2} / \mathrm{F}$ and $\gamma=0$, if not otherwise stated, namely the material parameters that have been reported for the $\mathrm{Hf}_{0.5} \mathrm{Zr}_{0.5} \mathrm{O}_{2}-\mathrm{Ta}_{2} \mathrm{O}_{5}$ MFIM system in. ${ }^{13}$

Fig.2(a) reports the maximum eigenvalue $\sigma_{\max }$ of the Jacobian for all $P_{i}=0$ versus the coupling factor $k$ for either MFMIM or MFIM structures with an area $\mathrm{A}=2500 \mathrm{~nm}^{2}$, and for different combinations of $n_{D}$ and $d$. As it can be seen the MFIM capacitor can achieve NC stabilisation for smaller $k$ values compared to the MFMIM system, and it has a much weaker sensitivity to the increase of $n_{D}$. The substantial difference in the NC stabilisation of 


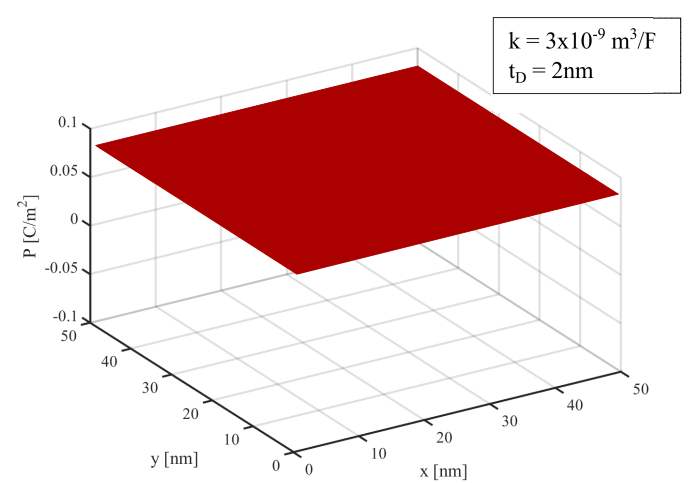

c

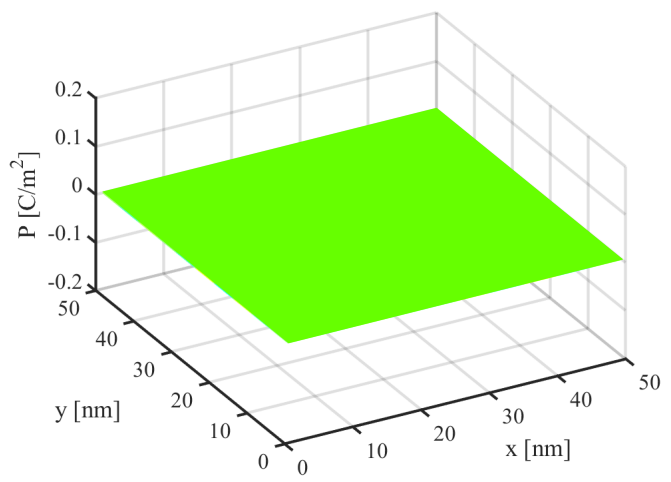

b

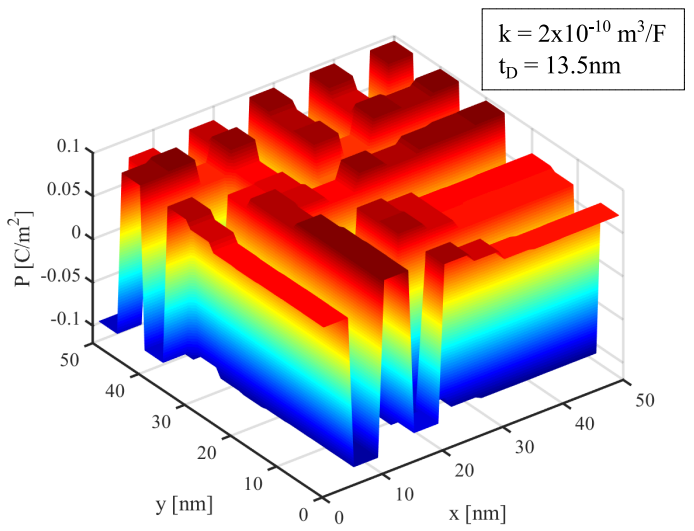

d

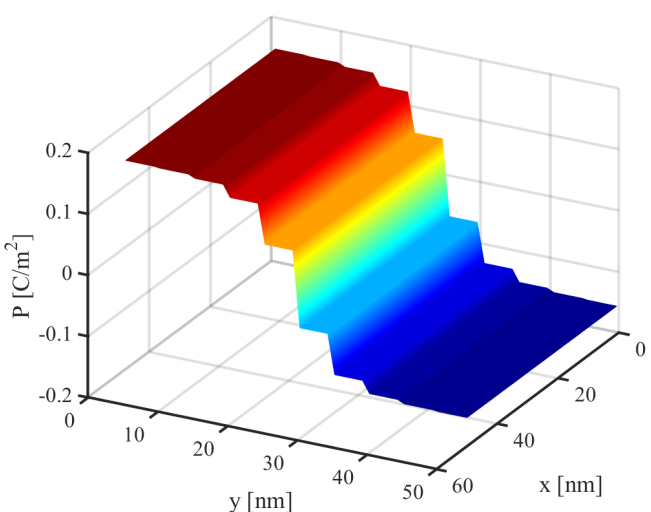

Figure 3: Ferroelectric domain patterns for MFIM and MFMIM capacitors. Steady-state domain configuration at $V_{T}=0$ V. a. MFIM system: small $t_{D}$ and high $k$ value that do not correspond to a stable NC operation, see triangle in Fig.2(b). b. MFIM system: large $t_{D}$ and small $k$ value that do not correspond to a stable NC operation, see star in Fig.2(b). c. MFIM system with $t_{D}=13.5 \mathrm{~nm}, t_{F}=11.6 \mathrm{~nm}$ and $k=2 \times 10^{-9} \mathrm{~m}^{3} / \mathrm{F}$, which correspond to a stable NC operation, see square in Fig.2(b). d. MFMIM capacitor having the same material and device parameters as the MFIM in $\mathbf{c}$.

MFMIM and MFIM systems for large $n_{D}$ is better illustrated by Fig.2(b), showing that for the MFMIM system the $k$ value required for NC stabilisation increases proportionally to $n_{D}$ and thus to the device areas. This makes NC stabilisation practically impossible for MFMIM systems having areas as those used in recent experiments. ${ }^{14-16}$

Fig.2(c)(d) focus on the MFIM system and report respectively the numerically calculated $\sigma_{\max }$ of the Jacobian matrix (for all $P_{i}=0$ ) for different $t_{D}$ and at fixed $t_{F}$, and the design regions for a stable NC operation of a MFIM structure in the $t_{D}-k$ plane and for $n_{D}=100$. As it can be seen the NC operation is not possible for too thin oxides, because the necessary condition $\left(1 / C_{0}\right)>2|\alpha| t_{F}$ is not fulfilled and, for any $t_{D}$ satisfying the above condition, we have a minimum $k$ value necessary for stabilisation. For $t_{D}$ larger than about $10 \mathrm{~nm}$ the $k$ for NC stabilisation becomes independent of $t_{D}$. This occurs because, while at small $t_{D}$ the potential $V_{D}$ at the ferroelectric-dielectric interface and the depolarisation energy $U_{d e p}$ decrease by scaling $t_{D}$, at large $t_{D}$ the $U_{d e p}$ becomes insensitive to $t_{D}$.

According to the empirical formula for the NC stable operation of a one-dimensional and periodic MFIM system proposed in Eq.15 of, ${ }^{18}$ the $t_{D}$ independent $k$ value necessary for $\mathrm{NC}$ operation is $k=1.2 \cdot 10^{9}\left[\mathrm{~m}^{3} / \mathrm{F}\right]$ for $t_{F}=11.6 \mathrm{~nm}$, and $k=2.1 \cdot 10^{9}\left[\mathrm{~m}^{3} / \mathrm{F}\right]$ for $t_{F}=20 \mathrm{~nm}$. These $k$ values are about two times larger than the values in Fig.2(d) obtained for the two-dimensional ferroelectric domain arrangement studied in this work. In more general terms we found that, while the qualitative trends obtained from our 3D analysis are similar to those predicted by the Eq. 15 of, ${ }^{18}$ the regions for NC stabilization identified by our results are larger. For example our 3D results suggest that, for a given couple $\left(t_{D}, t_{F}\right)$, a smaller $k$ is sufficient for stabilization and, for a given $\left(t_{D}, k\right)$, the system is NC stable up to larger $t_{F}$ values.

While Fig.2 illustrates the design space for a stable NC operation, it is also insightful to inspect the steady-state configuration of domains obtained by solving the LGD dynamic equations. In this respect, Fig.3(a) reports the steady-state domain configuration at $V_{T}=0$ for a MFIM system corresponding to the triangle symbol in Fig.2(d), namely to a system where the condition $\left(1 / C_{0}\right)>2|\alpha| t_{F}$ necessary for $\mathrm{NC}$ stabilisation is not fulfilled. As it 
can be seen the MFIM evolves so as to minimise the domain wall energy, whose minimum value is achieved by having all the domains with a positive polarisation. This steady-state polarisation pattern resembles the pattern of a MFM system, which the MFIM capacitor in fact approximates when $t_{D}$ and $U_{d e p}$ become very small. Fig.3(b), instead, illustrates the case corresponding to the star symbol in Fig.2(d), namely to a system where the condition $\left(1 / C_{0}\right)>2|\alpha| t_{F}$ is fulfilled, but the domain wall constant $k$ is too small for the NC stabilisation. In this case the system tends to minimise the depolarisation energy by having domains with different polarisations, even if this implies a larger domain wall energy compared to the pattern in Fig.3(a). Fig.3(c),(d) illustrate the steadystate domain configuration at $V_{T}=0$ for a MFIM with $k=2 \cdot 10^{-9} \mathrm{~m}^{3} / \mathrm{F}$ (square symbol in Fig.2(d)), and for the counterpart MFMIM. Consistently with Fig.2(d), the steady-state condition for the MFIM system corresponds to all $P_{i}=0$. The MFMIM, instead, is not stable for all $P_{i}=0$, and therefore it evolves to a configuration corresponding to $P_{A V}=\left(\sum_{i=1}^{n_{D}} P_{i}\right) / n_{D} \simeq 0$.

Fig.3(c) and (d) demonstrate that the crucial difference between MFMIM and MFIM systems is that the depolarisation energy of the MFMIM system at $V_{T}=0$ is zero if $P_{A V}$ is zero (see Eq.7). Hence if the MFMIM is initialised with all $P_{i}=0$, it gets destabilised along trajectories having $P_{A V} \simeq 0$ and thus $U_{d e p} \simeq 0$, which is confirmed by the steady-state configuration in Fig.3(d). The same trajectories are precluded in the MFIM system because the corresponding $U_{d e p}$ in Eq.7 is not at all zero, hence it is the form of the $U_{d e p}$ which makes the NC stabilisation possible in MFIM capacitors.

The analysis developed in this paper and the results presented in this section assumed that the leakage current through the oxides is small enough to not influence the $\mathrm{NC}$ stabilization. As already recognized in, ${ }^{24,25}$ in a MFMIM structure the presence of a non negligible leakage essentially precludes the NC stabilization.

\section{Comparison with experimental results}

As a validation of our modelling approach we now illustrate a systematic comparison with recent experiments reported for an $\mathrm{Hf}_{0.5} \mathrm{Zr}_{0.5} \mathrm{O}_{2}$ based MFIM structure. ${ }^{12,13}$ The simulations account for the presence of a fixed charge $Q_{D F}=0.15 \mathrm{C} / \mathrm{cm}^{2}$ at the interface between $\mathrm{Hf}_{0.5} \mathrm{Zr}_{0.5} \mathrm{O}_{2}$ and $\mathrm{Ta}_{2} \mathrm{O}_{5}$, which results in the fact that the ferroelectric is biased in the negative polarisation branch for $V_{T}=0 \mathrm{~V} .{ }^{12}$ Simulations correspond to a domain size of $d=5 \mathrm{~nm}$ and a domain number $n_{D}=100$, and we verified that results are insensitive to any further $n_{D}$ increase. The pulse width of the trapezoidal input waveform $V_{T}(t)$ is set to $1 \mu \mathrm{s}$ (if not otherwise stated), which is small enough to make the ferroelectric time constants practically negligible for the small resistivity value $\rho=0.5 \mathrm{~m} \Omega \cdot \mathrm{m}$ employed in these simulations.

Fig.4(a) reports the charge $Q=P+\varepsilon_{F} \varepsilon_{0} E_{F}+Q_{D F}$ versus the top value $V_{\max }$ of the trapezoidal voltage waveform applied across the $\mathrm{Hf}_{0.5} \mathrm{Zr}_{0.5} \mathrm{O}_{2}-\mathrm{Ta}_{2} \mathrm{O}_{5}$ capacitor, and shows a good agreement between simulations and experiments. Fig.4(b) illustrates the simulated waveforms for the ferroelectric field, $E_{F}$, and the total ferroelectric polarizarion, $P_{T}=\mathrm{P}+\varepsilon_{F} \varepsilon_{0} E_{F}$, produced by trapezoidal input $V_{T}$ and for three $V_{T}$ amplitudes. By using the $E_{F}$ and $P_{T}$ values observed in Fig.4(b), we obtained the charge versus ferroelectric field curves reported in Fig.4(c)(d) respectively for the $\mathrm{Hf}_{0.5} \mathrm{Zr}_{0.5} \mathrm{O}_{2}-\mathrm{Ta}_{2} \mathrm{O}_{5}$ and $\mathrm{Hf}_{0.5} \mathrm{Zr}_{0.5} \mathrm{O}_{2}-\mathrm{Al}_{2} \mathrm{O}_{3}$ capacitor. As it can be seen simulations nicely reproduce the fact that, for the experimental conditions at study, the ferroelectric layer can be operated in the NC operation region, which is the physical origin of the change of slope in the $Q$ versus $V_{\max }$ plot of Fig.4(b). We also verified that, as long as the NC stabilization is guaranteed, different $t_{D}$ values still result in the same $P_{T}$ versus $E_{F}$ curves for the quasi-static NC operation explored in this work.

From the charge versus $V_{T}$ plots as in Fig.4(a) we numerically calculated the capacitance $C_{T}=\left(\partial Q / \partial V_{T}\right)$ in the NC stabilized region and compared to results the simple analytical expression $C_{T}=C_{D} \cdot\left[\left|C_{F, 0}\right| /\left(\left|C_{F, 0}\right|-C_{D}\right)\right]$, with $C_{F, 0}=\left[1 /\left(2 \alpha t_{F}\right)+\varepsilon_{F} / t_{F}\right]$ being the zero field ferroelectric capacitance. This analysis showed that, while the numerically calculated $C_{T}$ is quite bias dependent even inside the $\mathrm{NC}$ region, the analytical expression is in very close agreement with the maximum $C_{T}$. Because the term $\left[\left|C_{F, 0}\right| /\left(\left|C_{F, 0}\right|-C_{D}\right)\right]$ can be seen as an enhancement factor of $C_{T}$ with respect to $C_{D}$, the analytical expression allows one to easily estimate the capacitance enhancement from the dielectric and ferroelectric parameters $\varepsilon_{D}, t_{D}, \alpha, \varepsilon_{F}, t_{F}$.

We also developed a model to study the influence of traps at the ferroelectric-dielectric interface, according to a simple kinetic equation for the trap occupation that we solved self-consistently with the LGD equations, as discussed in detail in the Supplementary Section S6. Fig.4(e) illustrates that traps are assumed to exchange electrons via tunneling with the bottom metal contact. While the bias independent rate $e_{n 0}$ could be described 
by models similar to those used for border traps in MOS transistors, ${ }^{26,27}$ such a quantitative description of the emission rates goes beyond the scope of the present work, where we investigate only the qualitative features induced by traps and, to this purpose, we consider $e_{n 0}$ as a free parameter in the comparison to experiments. In this respect, Fig.4(f) illustrates experiments and simulations for the charge versus ferroelectric field obtained for different pulse widths of the trapezoidal input waveform, where simulations correspond to a uniform density $N_{T}=7.5 \times 10^{12} \mathrm{eV}^{-1} \mathrm{~cm}^{-2}$ of acceptor type traps. As it can be seen, by using $e_{n 0}=5.0 \cdot 10^{4} \mathrm{~s}^{-1}$ the simulations can reproduce quite well the influence of the pulse width on the $Q$ versus $E_{F}$ curves observed in experiments. The influence of traps on the stability conditions of a MFIM system is further addressed in the Supplementary Section S6.

In summary, we presented a methodology to investigate a possible stable NC operation in ferroelectric capacitors based on the LGD dynamic equations and duly accounting for the three-dimensional nature of the problem. From the Jacobian matrix of the LGD equations we derived analytical or semi-analytical stability conditions, that clarified important differences between a MFIM and a MFMIM system. Our analysis is consistent with the fact that a stable NC operation has been observed in MFIM systems but not in MFMIM systems, and suggests MFMIM capacitors or capacitors externally connected to a MOSFET are inherently unsuitable to study the stable NC operation.

A systematic comparison with recent experiments in MFIM capacitors provides convincing evidence that the $\mathrm{NC}$ operation of the ferroelectric $\mathrm{Hf}_{0.5} \mathrm{Zr}_{0.5} \mathrm{O}_{2}$ can nicely explain experimental data. The critical role of interface traps emphasizes the importance of the quality of the ferroelectric-dielectric interface in the NC operation of ferroelectric capacitors and transistors.

We conclude by remarking that, while in a robustly NC stabilized system domains tend to move together thus resulting in a fairly $1 \mathrm{D}$ electrostatics, we verified that the electrostatics becomes strongly $3 \mathrm{D}$ when domain nucleation occurs and the system becomes hysteretic. The methodology for the dynamics of ferroelectric domain developed in this paper is thus expected to be important also for the analysis of a transient and possibly hysteretic NC operation, as well as for the investigation of Ferroelectric Tunnelling Junctions to be used either as non volatile memories or as memristors for neuromorphic computing applications. ${ }^{28}$ 
a

c
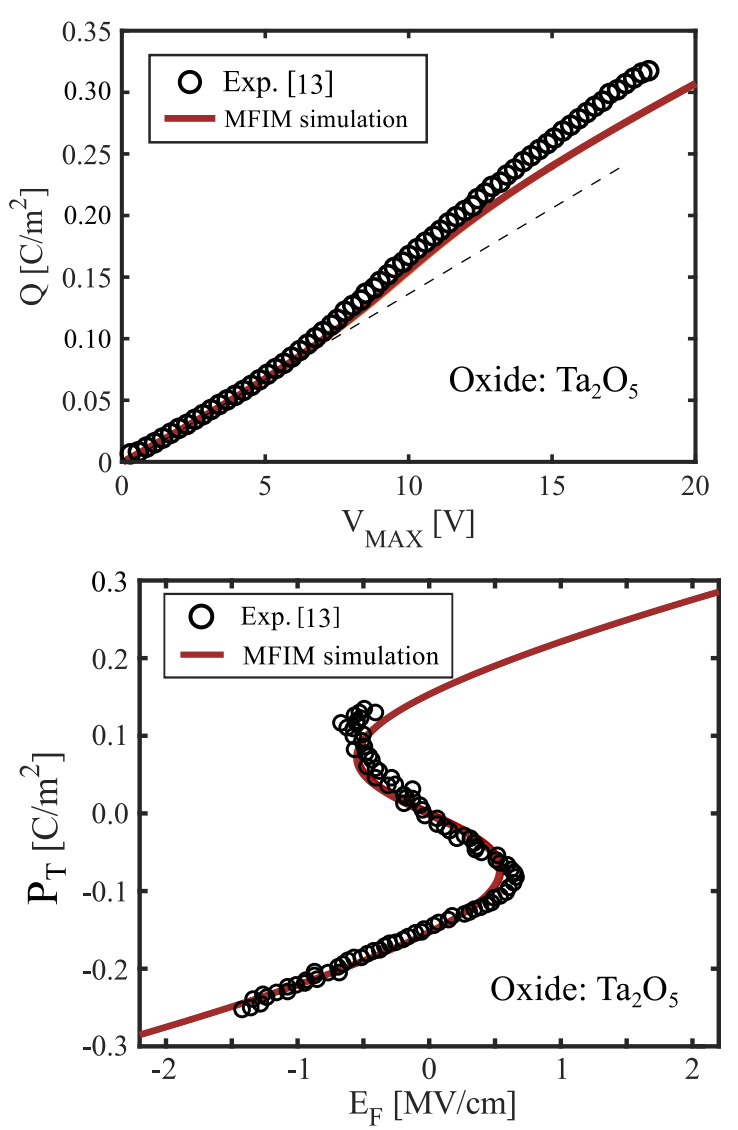

e

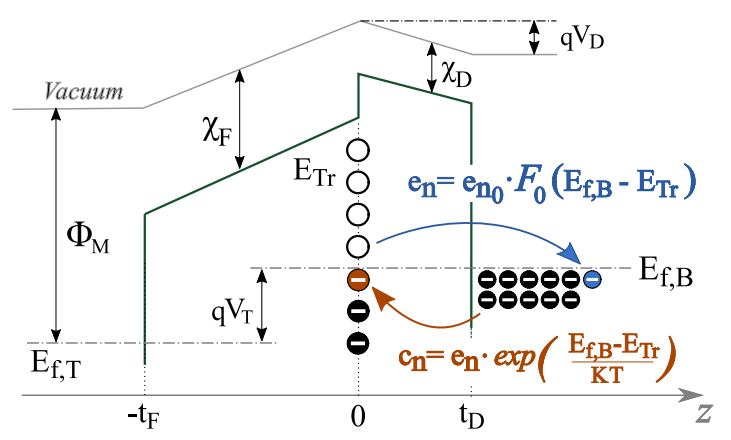

b

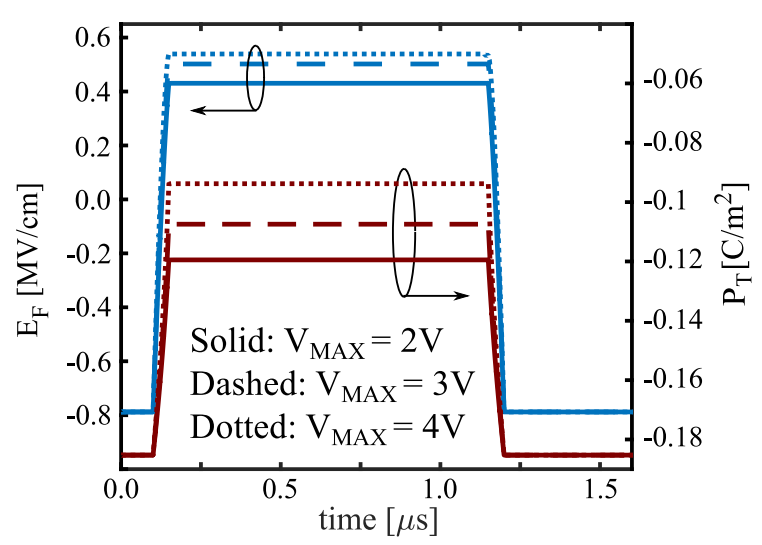

d

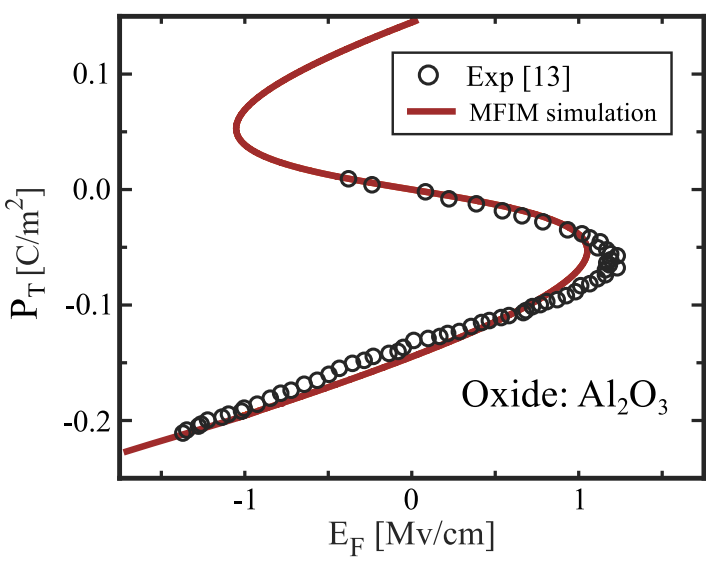

f

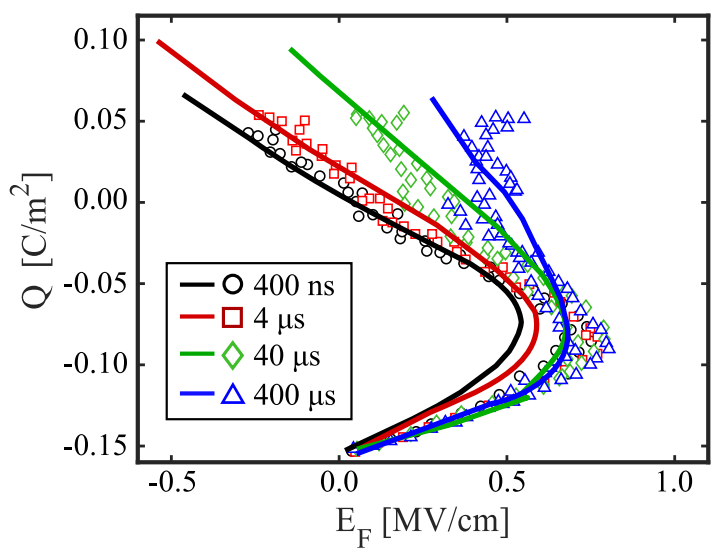

Figure 4: Comparison between simulations and experiments. Measurements (symbols) and simulations (lines) for the MFIM structures in. ${ }^{12,13}$ For the $\mathrm{Hf}_{0.5} \mathrm{Zr}_{0.5} \mathrm{O}_{2}-\mathrm{Ta}_{2} \mathrm{O}_{5}$ capacitor the simulation parameters are $\varepsilon_{F}=33, \varepsilon_{D}=23.48, t_{F}=11.6 \mathrm{~nm}, t_{D}=13.5 \mathrm{~nm}$, $\alpha=-4.6 \cdot 10^{8} \mathrm{~m} / \mathrm{F}, \beta=9.8 \cdot 10^{9} \mathrm{~m}^{5} / \mathrm{C}^{2} / \mathrm{F}$, while for the $\mathrm{Hf}_{0.5} \mathrm{Zr}_{0.5} \mathrm{O}_{2}-\mathrm{Al}_{2} \mathrm{O}_{3}$ system the parameters are $\varepsilon_{D}=8, t_{F}=7.7 \mathrm{~nm}, t_{D}=4 \mathrm{~nm}$, $\alpha=-9.45 \cdot 10^{8} \mathrm{~m} / \mathrm{F}$ and $\beta=2.25 \cdot 10^{10} \mathrm{~m}^{5} / \mathrm{C}^{2} / \mathrm{F} ;{ }^{12,13}$ for both capacitors we used $\rho=0.5 \mathrm{~m} \Omega \cdot \mathrm{m}$ and $k=2 \cdot 10^{-9} \mathrm{~m}^{3} / \mathrm{F} \mathrm{m}$. a. Reversibly stored and released charge, $Q$, versus the top value $V_{M A X}$ of the trapezoidal voltage waveform across the capacitor. b. Simulated ferroelectric field and charge versus time produced by a trapezoidal input $V_{T}$ with a pulse width of $1 \mu$ s and for different $V_{T}$ amplitudes. c. Polarisation versus ferroelectric electric field for the $\mathrm{Hf}_{0.5} \mathrm{Zr}_{0.5} \mathrm{O}_{2}-\mathrm{Ta}_{2} \mathrm{O}_{5}$ MFIM capacitor. d. Polarisation versus ferroelectric electric field for the $\mathrm{Hf}_{0.5} \mathrm{Zr}_{0.5} \mathrm{O}_{2}-\mathrm{Al}_{2} \mathrm{O}_{3}$ capacitor. e. Sketch of the band structure of the MFIM device with representation of the emission and capture mechanisms. f. Simulated charge versus ferroelectric $E_{F}$ curves for different pulse widths of the input signal and fixed density $N_{T}=7.5^{12} \mathrm{eV}^{-1} \mathrm{~cm}^{-2}$ of acceptor type interface traps with a uniform energy distribution. In these simulations the emission rate is $e_{n 0}=5 \times 10^{4} \mathrm{~s}^{-1}$, the metal gate work-function is $\Phi_{M}=4.05 \mathrm{eV}$, and the electron affinity is $\chi_{F}=2.2 \mathrm{eV}$ for $\mathrm{Hf}_{0.5} \mathrm{Zr}_{0.5} \mathrm{O}_{2}$ and $\chi_{D}=3.2 \mathrm{eV}$ for $\mathrm{Ta}_{2} \mathrm{O}_{5} .{ }^{29}$ 


\section{References}

${ }^{1}$ S. Salahuddin and S. Datta, "Use of Negative Capacitance to Provide Voltage Amplification for Low Power Nanoscale Devices," Nano Letters, vol. 8, no. 2, 2008. doi: 10.1021/nl071804g

2 A. I. Khan, "On the Microscopic Origin of Negative Capacitance in Ferroelectric Materials: A Toy Model," in 2018 IEEE International Electron Devices Meeting (IEDM), 2018. doi: 10.1109/IEDM.2018.8614574. ISSN 2156-017X pp. 9.3.1-9.3.4.

3 J. C. Wong and S. Salahuddin, "Negative Capacitance Transistors," Proceedings of the IEEE, vol. 107, no. 1, pp. 49-62, 2019.

${ }^{4}$ T. N. Theis and P. M. Solomon, "In Quest of the Next Switch: Prospects for Greatly Reduced Power Dissipation in a Successor to the Silicon Field-Effect Transistor," Proceedings of the IEEE, vol. 98, no. 12, pp. 2005-2014, 2010 .

${ }^{5}$ A. C. Seabaugh and Q. Zhang, "Low-Voltage Tunnel Transistors for Beyond CMOS Logic," Proceedings of the IEEE, vol. 98, no. 12, pp. 2095-2110, 2010. doi: 10.1109/JPROC.2010.2070470

${ }^{6}$ Z. Krivokapic, U. Rana, R. Galatage, A. Razavieh, A. Aziz, J.Liu, J.Shi, H. Kim, R. Sporer, C. Serrao, A.Busquet, P. Polakowski, J. Mülle, W. Kleemeier, A. Jacob, D. Brown, A. Knorr, R. Carter, and S. Banna, GLOBALFOUNDRIES, "14nm Ferroelectric FinFET Technology with Steep Subthreshold Slope for Ultra Low Power Applications," in 2017 IEEE International Electron Devices Meeting (IEDM), 2017. doi: 10.1109/IEDM.2017.8268393. ISSN 2156-017X pp. 357-360.

${ }^{7}$ D. Kwon, Y. Liao, Y. Lin, J. P. Duarte, K. Chatterjee, A. J. Tan, A. K. Yadav, C. Hu, Z. Krivokapic, and S. Salahuddin, "Response Speed of Negative Capacitance FinFETs," in 2018 IEEE Symposium on VLSI Technology, 2018. doi: 10.1109/VLSIT.2018.8510626. ISSN 2158-9682 pp. 49-50.

${ }^{8}$ T. Rollo and D. Esseni, "Energy Minimization and Kirchhoff's Laws in Negative Capacitance Ferroelectric Capacitors and MOSFETs," IEEE Electron Device Letters, vol. 38, no. 6, pp. 814-817, 2017. doi: 10.1109/LED.2017.2691002

${ }^{9}$ T. Rollo and D. Esseni, "New Design Perspective for Ferroelectric NC-FETs," IEEE Electron Device Letters, vol. 39, no. 4, pp. 603-606, 2018. doi: 10.1109/LED.2018.2795026

${ }^{10}$ T. Rollo and D. Esseni, "Influence of Interface Traps on Ferroelectric NC-FETs," IEEE Electron Device Letters, vol. 39, no. 7, pp. 1100-1103, 2018. doi: 10.1109/LED.2018.2842087

${ }^{11}$ M. A. Alam, M. Si, and P. D. Ye, "A critical review of recent progress on negative capacitance field-effect transistors," Applied Physics Letters, vol. 114, no. 9, p. 090401, 2019. doi: 10.1063/1.5092684. [Online]. Available: https://doi.org/10.1063/1.5092684

${ }^{12}$ M. Hoffmann, B. Max, T. Mittmann, U. Schroeder, S. Slesazeck, and T. Mikolajick, "Demonstration of Highspeed Hysteresis-free Negative Capacitance in Ferroelectric $\mathrm{Hf}_{0.5} \mathrm{Zr}_{0.5} \mathrm{O}_{2}$," in 2018 IEEE International Electron Devices Meeting (IEDM), 2018. doi: 10.1109/IEDM.2018.8614677. ISSN 2156-017X pp. 31.6.1-31.6.4.

${ }^{13}$ M. Hoffmann, F. P. G. Fengler, M. Herzig, T. Mittmann, B. Max, U. Schroeder, R. Negrea, P. Lucian, S. Slesazeck, and T. Mikolajick, "Unveiling the double-well energy landscape in a ferroelectric layer," Nature, vol. 565, pp. 464-467, 2019. doi: 10.1038/s41586-018-0854-z. [Online]. Available: https://doi.org/10.1038/s41586-018-0854-Z

${ }^{14}$ Z. Liu, M. A. Bhuiyan, and T. P. Ma, "A Critical Examination of 'Quasi-Static Negative Capacitance' (QSNC) theory," in 2018 IEEE International Electron Devices Meeting (IEDM), 2018. doi: 10.1109/IEDM.2018.8614614. ISSN 2156-017X pp. 31.2.1-31.2.4.

${ }^{15} \mathrm{X}$. Li and A. Toriumi, "Direct relationship between sub- $60 \mathrm{mV} / \mathrm{dec}$ subthreshold swing and internal potential instability in MOSFET externally connected to ferroelectric capacitor," in 2018 IEEE International Electron Devices Meeting (IEDM), 2018. ISSN 2156-017X pp. 31.3.1-31.3.4. 
${ }^{16}$ H. Wang, M. Yang, Q. Huang, K. Zhu, Y. Zhao, Z. Liang, C. Chen, Z. Wang, Y. Zhong, X. Zhang, and R. Huang, "New Insights into the Physical Origin of Negative Capacitance and Hysteresis in NCFETs," in 2018 IEEE International Electron Devices Meeting (IEDM), 2018. doi: 10.1109/IEDM.2018.8614504. ISSN 2156-017X pp. 31.1.1-31.1.4.

${ }^{17}$ J. Van Houdt and P. Roussel, "Physical Model for the Steep Subthreshold Slope in Ferroelectric FETs," IEEE Electron Device Letters, vol. 39, no. 6, pp. 877-880, 2018. doi: 10.1109/LED.2018.2829604

${ }^{18}$ M. Hoffmann, M. Pešić, S. Slesazeck, U. Schroeder, and T. Mikolajick, "On the stabilization of ferroelectric negative capacitance in nanoscale devices," Nanoscale, vol. 10, pp. 10891-10899, 2018. doi: 10.1039/C8NR02752H. [Online]. Available: http://dx.doi.org/10.1039/C8NR02752H

${ }^{19}$ T. Rollo, F. Blanchini, G. Giordano, R. Specogna, and D. Esseni, "Revised analysis of negative capacitance in ferrolectric-insulator capacitors: analytical and numerical results, physical insight, comparison to experiments. ," in 2019 IEEE International Electron Devices Meeting (IEDM), 2019, pp. 7.2.1-7.2.4.

${ }^{20} \mathrm{C}$. H. Woo and Y. Zheng, "Depolarization in modeling nano-scale ferroelectrics using the Landau free energy functional," Applied Physics A, p. 59-63, 2008. doi: 10.1007/s00339-007-4355-4

${ }^{21}$ E. M. Purcell, Electricity and Magnetism. Oxford University, 2013. ISBN 0-89871-465-6

${ }^{22}$ D. G. Luenberger, Introduction To Dynamic Systems. John Wiley \& Sons, 1979. ISBN 10:0471025941

${ }^{23}$ M. Fiedler, “Algebraic connectivity of graphs," Czechoslovak Mathematical Journal, vol. 23, no. 2, pp. 298-305, 1973. [Online]. Available: http://eudml.org/doc/12723

${ }^{24}$ A. I. Khan, U. Radhakrishna, S. Salahuddin, and D. Antoniadis, "Work function engineering for performance improvement in leaky negative capacitance fets," IEEE Electron Device Letters, vol. 38, no. 9, pp. 1335-1338, 2017. doi: 10.1109/LED.2017.2733382

${ }^{25}$ A. I. Khan, U. Radhakrishna, K. Chatterjee, S. Salahuddin, and D. A. Antoniadis, "Negative capacitance behavior in a leaky ferroelectric," IEEE Transactions on Electron Devices, vol. 63, no. 11, pp. 4416-4422, 2016.

${ }^{26}$ A. Palma, A. Godoy, J. A. Jiménez-Tejada, J. E. Carceller, and J. A. López-Villanueva, "Quantum two-dimensional calculation of time constants of random telegraph signals in metal-oxide-semiconductor structures," Phys. Rev. B, vol. 56, pp. 9565-9574, 1997. doi: 10.1103/PhysRevB.56.9565. [Online]. Available: https://link.aps.org/doi/10.1103/PhysRevB.56.9565

${ }^{27}$ F. Jiménez-Molinos, F. Gámiz, A. Palma, P. Cartujo, and J. A. López-Villanueva, "Direct and trap-assisted elastic tunneling through ultrathin gate oxides," Journal of Applied Physics, vol. 91, no. 8, pp. 5116-5124, 2002. doi: $10.1063 / 1.1461062$

${ }^{28}$ B. Sören, G. Julie, L. Gwendal, X. Bin, L. Nicolas, F. Stéphane, G. Stéphanie, C. Cécile, G. Karin, X. Stéphane, T. Jean, B. Laurent, B. Manuel, B. Agnès, S. Sylvain, and G. Vincent, "Learning through ferroelectric domain dynamics in solid-state synapses," Nature Communications, vol. 8, 2017. doi: 10.1038/ncomms14736

${ }^{29}$ B. C.-m. Lai, "Leakage Current Mechanism of Metal- $\mathrm{Ta}_{2} \mathrm{O}_{5}-$ Metal Capacitors for Memory Device Applications," Journal of The Electrochemical Society, vol. 146, p. 266, 1999. doi: 10.1149/1.1391597. [Online]. Available: http://doi.org/10.1149/1.1391597 


\section{Supplemental Information}

\section{S1 Electrostatic energy}

We here further discuss the expressions for $U_{E T}$ reported in Sec.2 for either a MFMIM or a MFIM capacitor. For both systems the starting point is the $U_{E T}$ definition in Eq.3.

As already mentioned in the main text, in the MFMIM capacitor the ferroelectric and dielectric fields are independent of $\bar{r}=(x, y)$ and we have $E_{F, T}=\left(C_{D} V_{T}-P_{A V}\right) /\left(t_{F} C_{0}\right), V_{D}=\left(C_{F} V_{T}+P_{A V}\right) / C_{0},{ }^{1}$ where $P_{A V}=\left(\sum_{j=1}^{n_{D}} P_{j}\right) / n_{D}$ is the average polarisation. By substituting the $E_{F, T}$ and $V_{D}$ expressions in Eq.3 we obtain

$$
\begin{aligned}
U_{E T} & =-\frac{V_{T}}{2} \frac{A}{d^{2}} \frac{C_{F}}{C_{0}}\left(C_{D} V_{T}-P_{A V}\right)-V_{T} \sum_{j=1}^{n_{D}} P_{j}+\frac{n_{D} P_{A V}}{2 C_{0}}\left(C_{F} V_{T}+P_{A V}\right) \\
& =\frac{n_{D} P_{A V}^{2}}{2 C_{0}}-\frac{C_{s} V_{T}^{2}}{2} n_{D}-n_{D} \frac{C_{D}}{C_{0}} V_{T} P_{A V}
\end{aligned}
$$

where the total device area is $A=n_{D} d^{2}$ and we grouped terms proportional to $P_{A V}^{2}, V_{T}^{2}$ and $V_{T} P_{A V}$. Eq.S1 coincides with Eq.6, with the depolarisation energy $U_{d e p}$ for the MFMIM system defined in Eq.7.

In the MFIM structure the calculation of the ferroelectic and dielectric field is a three-dimensional problem, that we can approach by recalling that $V_{T}$ and each spontaneous polarisation $P_{h}$ are the sources of the electric fields. Because the system is linear, the superposition of effects allows us to write

$$
E_{F, T}(\bar{r})=\sum_{h=1}^{n_{D}} P_{h} G_{F T, h}(\bar{r})+\frac{C_{D} V_{T}}{t_{F} C_{0}}
$$

where $G_{F T, h}(\bar{r})$ is the Green's function for the field $E_{F, T}(\bar{r})=E_{F, z}\left(\bar{r},-t_{F}\right)$ of a unitary charge per unit area in domain $h$, while the effect of $V_{T}$ is simply described by a capacitor divider. We can similarly write the potential $V_{D}(\bar{r})$ as

$$
V_{D}(\bar{r})=\sum_{h=1}^{n_{D}} P_{h} G_{D, h}(\bar{r})+\frac{C_{F}}{C_{0}} V_{T}
$$

where $G_{D, h}(\bar{r})$ is the Green's function for $V_{D}(\bar{r})$ of a unitary charge in domain $h$. By substituting Eqs.S2, S3 in Eq.3 we recognise that the calculation of $U_{E T}$ entails the evaluation of the integral of any $G_{D, h}$ over any domain area $D_{j}$, and of any $G_{F T, h}(\bar{r})$ over the device area $A$. Consequently we introduce the capacitances

$$
\frac{1}{C_{j, h}}=\frac{1}{d^{2}} \int_{D_{j}} G_{D, h}(\bar{r}) d \bar{r}
$$

as well as the adimensional coefficients

$$
B_{h}=\frac{\varepsilon_{0} \varepsilon_{F}}{d^{2}} \int_{A} G_{F T, h}(\bar{r}) d \bar{r}
$$

which allow us to write the $U_{E T}$ of the MFIM system as

$$
U_{E T}=\frac{1}{2} \sum_{j, h=1}^{n_{D}} \frac{P_{h} P_{j}}{C_{j, h}}-\frac{V_{T}}{2} \sum_{h}^{n_{D}} P_{h}\left(B_{h}+1+C_{D} / C_{0}\right)-\frac{C_{S} V_{T}^{2}}{2} n_{D} .
$$

By the definition of $C_{j, h}$ we see that by summing over $h$ we obtain the Green's function for $V_{D}(\bar{r})$ in domain $j$ of a uniform charge in the entire device area, so that we have

$$
\sum_{h=1}^{n_{D}} \frac{1}{C_{j, h}}=\frac{1}{C_{0}}, \quad \sum_{j, h=1}^{n_{D}} \frac{1}{C_{j, h}}=\frac{n_{D}}{C_{0}}
$$

where the second equality is due to the fact that the first sum is independent of the domain $j$. 
In consideration of the Neumann boundary conditions used for the electric field at the edges of the MFIM structure (see Supplementary Section S7), analytical derivations suggest and numerical calculations confirm that the $B_{h}$ defined in Eq.S5 evaluates to $B_{h} \simeq-C_{F} / C_{0}$ and it is independent of $h$ (not shown). By substituting $B_{h} \simeq-C_{F} / C_{0}$ in Eq.S6, we obtain the $U_{E T}$ expression for the MFIM capacitor given by Eq.6, with the depolarisation energy $U_{\text {dep }}$ defined in Eq.7.

For all the MIFM systems analysed in this work the capacitances $C_{j, h}$ were evaluated numerically and then used in all analyses. More details about the three-dimensional calculations of the capacitances are given in Supplementary Section S7.

\section{S2 Conditions for a stable NC operation in a MFMIM system}

For convenience of notation we here introduce $\theta=\left(t_{F} k\right) /(d w)$ and $\eta=1 / C_{0}$. Given the Laplacian matrix $\mathbf{L}$ and the all-one matrix $\mathbf{O}_{d e p}$, both having size $n_{D}$, we prove that

$$
\sigma_{\min }\left[\theta \mathbf{L}+\eta \frac{\mathbf{O}_{d e p}}{n_{D}}\right]=\min \left\{\eta, \theta \sigma_{1}(\mathbf{L})\right\}
$$

where $\sigma_{\min }[M]$ denotes the smallest eigenvalue of matrix $M$ and $\sigma_{1}(\mathbf{L})$ denotes the second smallest eigenvalue of the Laplacian $\mathbf{L}$.

The eigenvalues of matrix $\frac{\mathbf{o}_{d e p}}{n_{D}}$ are 1 , and 0 with multiplicity $n_{D}-1$. Since the graph is connected, $\mathbf{L}$ has an eigenvalue 0 with multiplicity 1 , while all the other eigenvalues $\sigma_{1}(\mathbf{L}) \leq \sigma_{2}(\mathbf{L}) \leq \cdots \leq \sigma_{n_{D}-1}(\mathbf{L})$ are real and positive. Matrix $\mathbf{L}$ and matrix $\frac{\mathbf{o}_{d e p}}{n_{D}}$ share the normalised eigenvector $v=\frac{\mathbf{1}_{n_{D}}}{\sqrt{n_{D}}}$ (where $\mathbf{1}_{n_{D}}$ denotes an all-one vector of size $n_{D}$ ), which is associated with the 0 eigenvalue of $\mathbf{L}$ (i.e. $\mathbf{L} v=0$ ) and with the 1 eigenvalue of $\frac{\mathbf{o}_{d e p}}{n_{D}}$ (i.e. $\left.\frac{\mathbf{o}_{\text {dep }}}{n_{D}} v=v\right)$.

Let us complement vector $v$ with matrix $V \in \mathbb{R}^{n_{D} \times n_{D}-1}$ to obtain an orthonormal matrix $T=\left[\begin{array}{ll}v & V\end{array}\right]$ (such that $T^{-1}=T^{\top}$ ). We can then apply this transformation to simultaneously diagonalise both matrices:

$$
\begin{aligned}
& T^{-1}\left(\theta \mathbf{L}+\eta \frac{\mathbf{O}_{d e p}}{n_{D}}\right) T=\left[\begin{array}{c}
v^{\top} \\
V^{\top}
\end{array}\right]\left(\theta \mathbf{L}+\eta \frac{\mathbf{O}_{d e p}}{n_{D}}\right)\left[\begin{array}{ll}
v & V
\end{array}\right] \\
& =\left[\begin{array}{cc}
0 & \mathbf{0}_{n_{D}-1}^{\top} \\
\mathbf{0}_{n_{D}-1} & \boldsymbol{\theta} \Lambda
\end{array}\right]+\left[\begin{array}{cc}
\eta & \mathbf{0}_{n_{D}-1}^{\top} \\
\mathbf{0}_{n_{D}-1} & \overline{\mathbf{0}}_{n_{D}-1}
\end{array}\right],
\end{aligned}
$$

where $\mathbf{0}_{n_{D}-1}$ denotes an all-zero vector of size $n_{D}-1, \overline{\mathbf{0}}_{n_{D}-1}$ an all-zero matrix of size $n_{D}-1$, and $\Lambda=\operatorname{diag}\left\{\sigma_{1}(\mathbf{L}), \ldots, \sigma_{n_{D}-1}(\mathbf{L})\right\}$ is the diagonal matrix carrying on the diagonal the nonzero eigenvalues of $\mathbf{L}$. It is then clear that the spectrum of $\theta \mathbf{L}+\eta \frac{\mathbf{O}_{\text {dep }}}{n_{D}}$ is the union of $\eta$ and of the nonzero scaled Laplacian eigenvalues $\theta \sigma_{1}(\mathbf{L}), \ldots, \theta \sigma_{n_{D}-1}(\mathbf{L})$. Therefore, the smallest eigenvalue is the minimum between $\eta$ and $\theta \sigma_{1}(\mathbf{L})$. By recalling that, for the Laplacian matrix corresponding to a rectangular grid, $\sigma_{1}(\mathbf{L})=\left[2 \sin \left(\pi /\left(2 \sqrt{n_{D}}\right)\right)\right]^{2}$, we obtain

$$
\sigma_{\min }\left[\frac{t_{F} k}{d w} \mathbf{L}+\frac{\mathbf{O}_{d e p}}{n_{D} C_{0}}\right]=\min \left\{\frac{1}{C_{0}}, \frac{t_{F} k}{d w}\left[2 \sin \left(\pi /\left(2 \sqrt{n_{D}}\right)\right)\right]^{2}\right\}
$$

that proves the stability condition in Eq.12.

\section{S3 Conditions for a stable NC operation in a MFIM system}

In the MFIM case the condition for a stable NC operation is given by Eq.11c, where $\mathbf{C}_{d e p}$ is defined in Eq.10. We argue that $\mathbf{C}_{d e p}$ has a large influence on the spectrum of the Jacobian matrix for the MFIM system, in fact $\mathbf{C}_{d e p}$ can modify all the eigenvalues of $\mathbf{C}_{d e p}+\mu \mathbf{L}$, where $\mu=\frac{t_{F} k}{d w}$. We have a necessary condition for stability in terms of the entries of $\mathbf{C}_{d e p}$. In fact, we notice that Eq.11c requires that the inequality $P^{\top}\left[\mathbf{C}_{d e p}+\mu \mathbf{L}\right] P>2|\alpha| t_{F}\|P\|^{2}>0$ be fulfilled for all nonzero $P$ vectors. We now take $P=\overline{1}^{\top}=\left[\begin{array}{lllll}1 & 1 & 1 & \ldots\end{array}\right]^{\top}$, such that $\|P\|^{2}=n_{D}$. Exploiting the fact that $\overline{1}^{\top} \mathbf{L} \overline{1}=0$, we obtain

$$
\frac{\overline{1}^{\top}\left[\mathbf{C}_{d e p}+\mu \mathbf{L}\right] \overline{1}}{n_{D}}=\frac{\overline{1}^{\top} \mathbf{C}_{d e p} \overline{1}}{n_{D}}=\frac{\sum_{i, j=1}^{n_{D}} \mathbf{C}_{d e p}(i, j)}{n_{D}}>2|\alpha| t_{F} .
$$


In view of the sum rule in Eq.S7, $\sum_{i, j=1}^{n_{D}} \mathbf{C}_{d e p}(i, j)=n_{D} / C_{0}$, the above inequality becomes

$$
\frac{1}{C_{0}}>2|\alpha| t_{F}
$$

Eq.S13 provides a necessary condition for a stable NC operation of the MFIM system.

\section{S4 Stability of the equilibrium for $V_{T} \neq 0$}

Here we discuss how the stability conditions discussed in Sec. 3 for the condition all $P_{i}=0$ and $V_{T}=0$, in fact ensure stability for any $V_{T}$ and corresponding $P_{i}$ configurations. All the dynamic systems in Eqs.8a, $8 \mathrm{~b}$ and $8 \mathrm{c}$ can be recast into the common form

$$
\frac{d P}{d t}=\mathbf{A} P+\mathbf{f}(P)+\mathbf{1} u,
$$

where $\mathbf{A}$ is a symmetric matrix, the vector function $\mathbf{f}(P)$ has polynomial components

$$
\mathbf{f}_{i}\left(P_{i}\right)=-\left(2 \alpha P_{i}+4 \beta P_{i}^{3}+6 \gamma P_{i}^{5}\right) / \rho,
$$

1 is a vector of all ones, and the constant $u$ can be either $u=\frac{1}{t_{F} \rho} V_{T}$ (in the MFM case) or $u=\frac{1}{t_{F} \rho}\left(C_{D} / C_{0}\right) V_{T}$ (in the MFMIM and MFIM cases). The Jacobian of the system in Eq.S14, computed at the generic equilibrium point $\bar{P}$, is

$$
\mathbf{J}(P)=\mathbf{A}-2 \alpha / \rho \mathbf{I}-\operatorname{diag}\left\{12 \beta \bar{P}_{i}^{2}+30 \bar{P}_{i}^{4}\right\} / \rho=\mathbf{J}(0)-\mathbf{D},
$$

where the symmetric matrix $\mathbf{J}(0)=\mathbf{A}-2 \alpha / \rho \mathbf{I}$ is the Jacobian computed at the equilibrium $\bar{P}_{i}=0$ (shown in Eqs.9a, 9b and 9c for the three different cases), while the diagonal matrix $\mathbf{D}=\operatorname{diag}\left\{12 \beta \bar{P}_{i}^{2}+30 \bar{P}_{i}^{4}\right\}$ has nonnegative diagonal entries because $\beta>0$ and $\gamma \geq 0$.

Now, assume that the symmetric Jacobian $\mathbf{J}(0)$ has negative eigenvalues or, equivalently, that it is negative definite: $x^{\top} \mathbf{J}(0) x<0$ for any vector $x \neq 0$. Then, also matrix $\mathbf{J}(P)$ is negative definite, because $x^{\top} \mathbf{J} x=x^{\top} \mathbf{J}(0) x-$ $x^{\top} \mathbf{D} x \leq x^{\top} \mathbf{J}(0) x<0$ for $x \neq 0$. Therefore, $\mathbf{J}(P)$ has negative eigenvalues.

Hence, to ensure the stability of all possible equilibria with a generic $V_{T}$, it is enough to guarantee the stability of the equilibrium $P_{i}=0$ corresponding to $V_{T}=0$, which is discussed in the main paper.

\section{S5 Statistical dispersion of the ferroelectric anisotropy constants}

In actual ferroelectric materials the anisotropy constants $\alpha, \beta$ and $\gamma$ may have domain to domain variations, and it has been argued also that, by accounting for such a statistical dispersion, simulations can improve the agreement with experiments in the analysis of metal-ferroelectric-metal systems. ${ }^{9}$

A straightforward extension of the stability analysis in Sec. 3 shows that, in the presence of a statistical dispersion of $\alpha$, stability conditions can still be expressed as in Eqs.11 if we substitute $|\alpha|$ in right hand side of the equations with $\alpha_{\max }$, here defined as the maximum $\left|\alpha_{i}\right|$, with $i=1,2 \ldots, n_{D}$ being an index identifying domains. If we consider a system consisting of many domains with an average value, $|\alpha|_{a v}$, of the $\left|\alpha_{i}\right|$ constants, the introduction of $\alpha_{\max }$ in the inequalities of Eqs.11 inherently implies a more stringent requirement for a stable NC operation compared to the same system with a negligible dispersion.

We introduced in our analysis a randomness of $\alpha_{i}, \beta_{i}$ constants (still keeping $\gamma=0$ ) and revisited some of the results in Fig. 2 for a MFIM system. In order to have a clear physical interpretation of the statistical dispersion in the system, our starting point is a statistical dispersion of the ferroelectric coercive field $E_{C}$. More precisely, we used a random generation of the coercive field $E_{C, i}$ in each domain by using a gaussian distribution of $E_{C}$ with a mean value $E_{C, a v}=0.54 \mathrm{MV} / \mathrm{cm}$ (corresponding to $\alpha=-4.6 \cdot 10^{8} \mathrm{~m} / \mathrm{F}$ and $\beta=9.8 \cdot 10^{9} \mathrm{~m}^{5} / \mathrm{C}^{2} / \mathrm{F}$, see the beginning of Sec.3), and for different standard deviations $E_{C, d e v}$. Then for each $E_{C, i}$ we calculated the corresponding $\alpha_{i}, \beta_{i}$ by using the analytical expressions $\alpha_{i}=-3 \sqrt{3} E_{c} /\left(2 P_{r}\right), \beta_{i}=3 \sqrt{3} E_{c} /\left(2 P_{r}^{3}\right)$, which hold for $\gamma=0{ }^{2}$ no dispersion of the remnant polarisation $P_{r}$ was considered in the calculations. The eigenvalues of the Jacobian matrices were calculated numerically in the condition of all $P_{i}=0$.

Fig.S1(a) shows an analysis similar to Fig.2(b) but for a fixed $\mathrm{Ta}_{2} \mathrm{O}_{5}$ thickness $t_{D}=13.5 \mathrm{~nm}$ and accounting for a statistical dispersion of $\alpha_{i}, \beta_{i}$. In the presence of such a statistical dispersion, each MFIM capacitor is a 


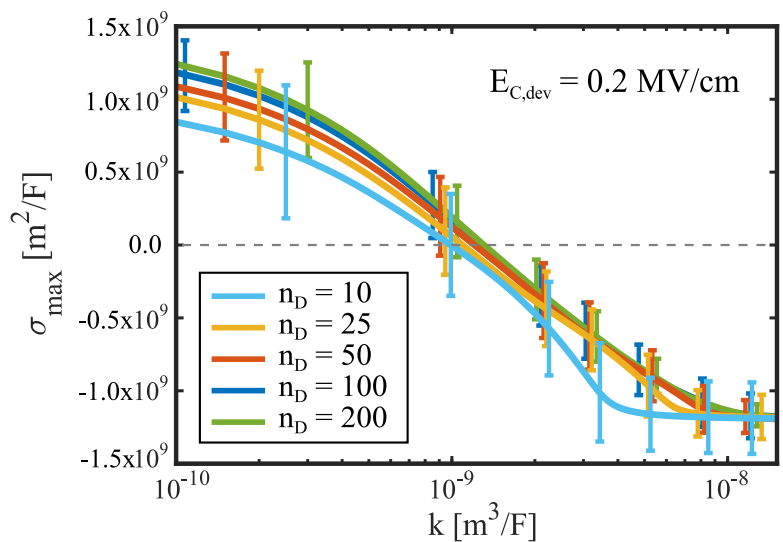

b

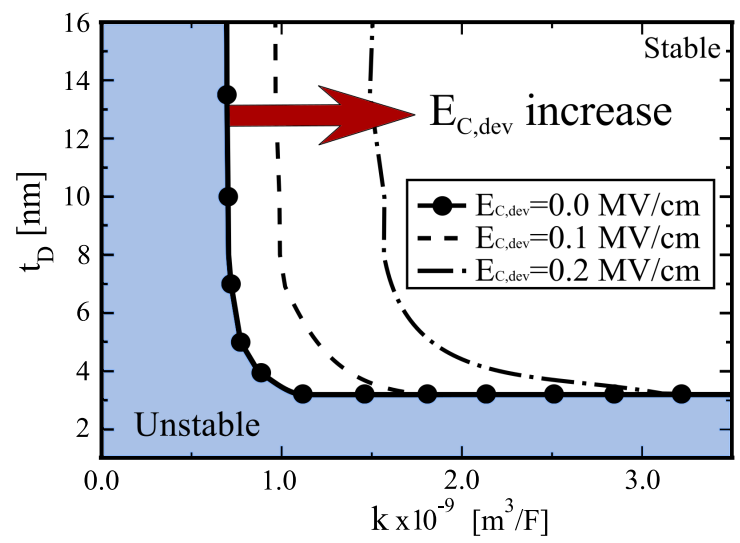

Figure S1: Eigenvalues of the Jacobian matrix and design space for stable NC operation in the presence of statistical dispersion of the anisotropy constants: a. Maximum eigenvalue $\sigma_{\max }$ of the Jacobian matrix for a $\mathrm{Hf}_{0.5} \mathrm{Zr}_{0.5} \mathrm{O}_{2}-\mathrm{Ta}_{2} \mathrm{O}_{5}$ MFIM structure (in the condition of all $P_{i}=0$ ) versus the domain wall coupling factor $k$. Calculations account for a statistical dispersion of $\alpha, \beta$ stemming from a standard deviation $E_{C, d e v}=0.2 \mathrm{MV} / \mathrm{cm}$ of the coercive field and correspond to 100 realizations of the MFIM structure. Lines show the $\sigma_{\max }$ averaged between the realizations, while the error bar symbols indicate the range between minimum and maximum $\sigma_{\max }$ among the different realizations at some $k$ values. Results are shown for different values of the domain number $n_{D}$, and all parameters other than $n_{D}$, $\alpha, \beta$ are the same as in calculations for $t_{D}=13.5 \mathrm{~nm}$ in Fig.2(c). b. Regions for stable NC operation for a MFIM structure in the $t_{D}$ versus $k$ plane and for different standard deviation $E_{C, d e v}$ of the coercive field. All parameters other than $n_{D}, \alpha, \beta$ are the same as in Fig. 2 for the case $t_{D}=13.5 \mathrm{~nm}, t_{F}=11.6 \mathrm{~nm}$. Area $\mathrm{A}=2500 \mathrm{~nm}^{2}$ and $n_{D}=100$.

statistical realization of a stochastic process and has a distinct set of eigenvalues of the Jacobian matrix, whose values depend also on the number $n_{D}$ of domains. Fig.S1(a) illustrates $\sigma_{\max }$ for a standard deviation $E_{C, d e v}=0.2$ $\mathrm{MV} / \mathrm{cm}$ of the coercive field, for 100 realizations and for different $n_{D}$ values. For each domain wall coupling factor $k$ the figure reports the $\sigma_{\max }$ averaged between the realizations (lines), and for some $k$ values the figure also shows the range between minimum and maximum $\sigma_{\max }$ among the different realizations. As it can be seen the average $\sigma_{\max }$ converges quite quickly by increasing $n_{D}$. Fig.S1(a) also shows that larger $k$ values are required for a stable NC operation for increasing $E_{C, d e v}$. This latter aspect is better illustrated in Fig.S1(b), that revisits the analysis in Fig.2(d) for $t_{F}=11.6 \mathrm{~nm}$ and for different standard deviation $E_{C, d e v}$ of the coercive field.

The results in Fig.S1(b) show a qualitative trend indicating that a statistical dispersion of the anisotropy constants results in more stringent requirements for the $k$ values necessary for a stable NC operation. In the presence of such a statistical dispersion, one should more appropriately discuss the probability of a stable NC operation in a given MFIM system, however such a statistical analysis of the stability properties goes beyond the scope of the present work.

\section{S6 Influence of interface traps on the stability conditions of a MFIM system}

The possible presence of traps at the ferroelectric-dielectric interface has an influence on NC stabilization because traps can partly screen the ferroelectric polarisation, which has an inherently destabilizing effect. Let us consider a number, $N_{E}$, of discrete trap levels in each domain, denote with $E_{T r, i}$ a trap energy in the domain $i$ (with $\operatorname{Tr}=1,2, \ldots, N_{E}$ and $i=1,2, \ldots, n_{D}$ ), and indicate with $Q_{T}$ the charge density per unit area due to traps. The present analysis is developed for a MFIM system, so that we can use a straightforward modification of Eq.8c and write the overall dynamic system as

$$
\begin{gathered}
t_{F} \rho \frac{d P_{i}}{d t}=\partial U_{L G D}-\frac{1}{2} \sum_{j=1}^{n_{D}}\left[\frac{1}{C_{i, j}}+\frac{1}{C_{j, i}}\right]\left(P_{j}+Q_{T, j}\right)+\frac{C_{D}}{C_{0}} V_{T}(t) \\
\frac{\partial n_{T r, i}}{\partial t}=c_{n}\left(N_{t}-n_{T r, i}\right)-e_{n} n_{T r, i}
\end{gathered}
$$

where $n_{T r, i}$ is the density of electrons trapped at energy $E_{T r}$ in domain $i$, while $N_{t}$ is the corresponding trap density. Here the emission rate is $e_{n}=e_{n 0} F_{0}\left[\left(E_{f, B}-E_{T r}\right) / K_{B} T\right]$, where $e_{n 0}$ is a bias independent rate and $F_{0}(\eta)$ is the FermiDirac equilibrium occupation function ${ }^{3}$. Hence $e_{n}$ depends on the external bias $V_{T}$ via the position of $E_{T r}$ with

\footnotetext{
${ }^{3}$ The Fermi-Dirac occupation function $F_{0}(\eta)$ is defined as $F_{0}(\eta)=1 /[1+\exp (\eta)]$.
} 
respect to $E_{f, B}$ and, in particular, it is equal to $e_{n 0}$ when $E_{T r}$ is at least a few $K_{B} T$ above $E_{f, B}$, whereas it decays exponentially for decreasing $E_{T r}$ when $E_{T r}$ is below $E_{f, B}$. The capture rate $c_{n}$ is expressed as $c_{n}=e_{n} \exp \left[\left(E_{f B}-\right.\right.$ $\left.\left.E_{T r}\right) / K_{B} T\right]$ so that, according to Eq.S15b, the steady-state occupation of traps is in thermodynamic equilibrium with $E_{f, B}$. The system in Eqs.S15 consists of $n_{D}+n_{D} \cdot N_{E}$ equations, hence the inclusion of traps substantially increases the size of the problem.

The $e_{n}, c_{n}$ expressions couple Eq.S15b to Eq.S15a because $E_{T r, i}$ depends on the average voltage $V_{D, i}$ at the ferroelectric-dielectric interface in the domain $i$. An explicit expression of the coupling between Eq.S15a and Eq.S15b is given by

$$
\begin{gathered}
Q_{T, i}=\Delta E \sum_{T r=1}^{N_{E}}(-q) n_{T r, i} \\
E_{T r, i}=\Phi_{M}-\left(\chi_{D}+E_{0}-i \Delta E\right)-q V_{D, i} \\
V_{D, i}=\sum_{j=1}^{n_{D}} \frac{\left(P_{j}+Q_{T, j}\right)}{C_{i, j}}+\frac{C_{F}}{C_{0}} V_{T}(t)
\end{gathered}
$$

where $\mathrm{E}_{T r, i}$ is the trap energy referred to $\mathrm{E}_{f B}$ (see Fig.4(e)). Here $\Phi_{M}$ and $\chi_{D}$ are respectively the metal gate work-function and dielectric electron affinity, $E_{0}$ is the depth into the dielectric bandgap of the deepest trap, $\Delta E$ is the energy step between the discrete trap levels, and Eq.S16a assumes traps are acceptor type.

Eqs.S15 and S16 summarize the model that we used for the simulations in Fig.4(f), and in this section we more formally study the stable NC operation by inspecting the sign of the Jacobian matrix of Eqs.S15. In this respect, Fig.S2(a) shows an analysis similar to Fig.2(c) but for a fixed $\mathrm{Ta}_{2} \mathrm{O}_{5}$ thickness $t_{D}=5 \mathrm{~nm}$ and different uniform trap densities $N_{T}$ at the $\mathrm{Hf}_{0.5} \mathrm{Zr}_{0.5} \mathrm{O}_{2}-\mathrm{Ta}_{2} \mathrm{O}_{5}$ interface. As it can be seen, by increasing $N_{T}$ the $\sigma_{\text {max }}$ at large $k$ values progressively increase and the system is eventually driven to instability for a large enough $N_{T}$. It is worth noting that the impact on $\sigma_{\max }$ of an increase of $N_{T}$ in Fig.S2(a) is qualitatively similar to the impact of a $t_{D}$ reduction in Fig.2(c). This can be intuitively explained by arguing that the trap capacitance $C_{i t}=q^{2} N_{T}$ goes in parallel to $C_{D}$, thus increasing the effective dielectric capacitance and eventually precluding the NC stabilization. This is confirmed by Fig.S2(b), showing that the charge versus ferroelectric field curves of the MFIM capacitor tend to deviate from the $\mathrm{NC}$ region when $N_{T}$ increases, with a behavior qualitatively similar to the results in Fig.4(f) where the pulse width of the trapezoidal input waveform was varied at fixed $N_{T}$.

a

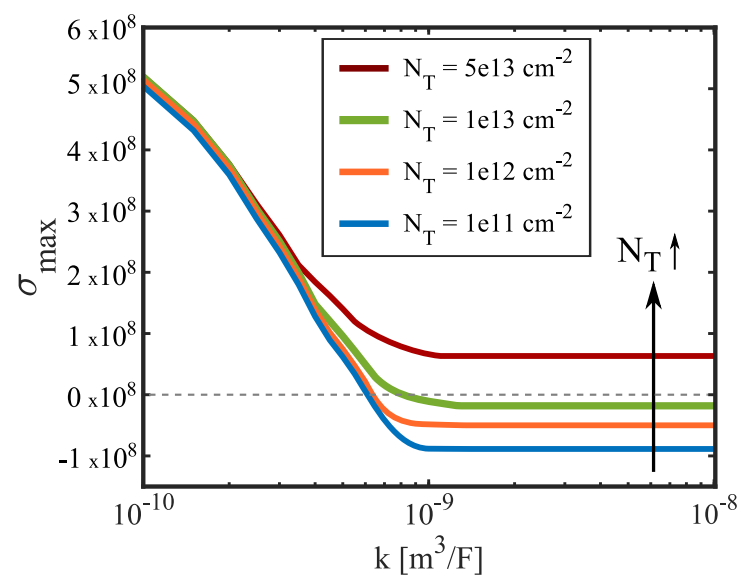

b

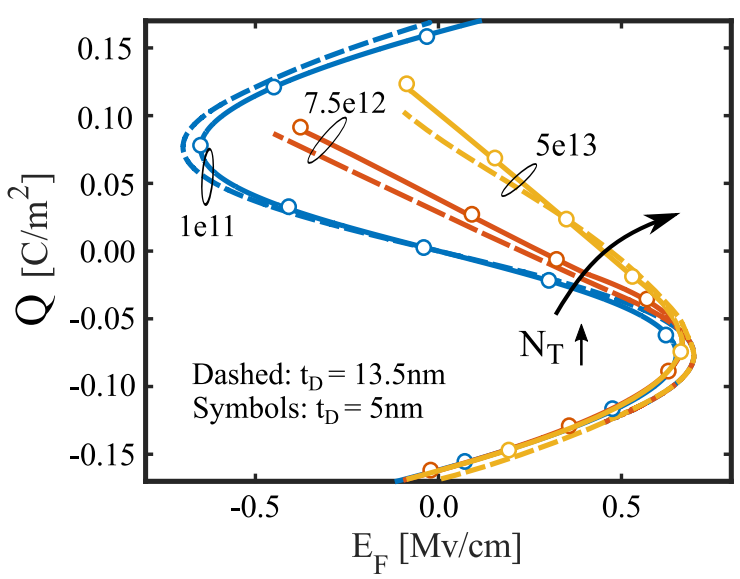

Figure S2: Eigenvalues of the Jacobian matrix and stable NC operation in the presence of interface traps: a. Maximum eigenvalue $\sigma_{\text {max }}$ of the Jacobian matrix (for all $P_{i}=0$ ) of a $\mathrm{Hf}_{0.5} \mathrm{Zr}_{0.5} \mathrm{O}_{2}-\mathrm{Ta}_{2} \mathrm{O}_{5}$ MFIM system versus the domain wall coupling factor $k$ for a uniform distribution acceptor type traps (extending for $3.2 \mathrm{eV}$ below the $\mathrm{Ta}_{2} \mathrm{O}_{5}$ conduction band minimum) and for an energy spacing $\Delta E=7.5$ $\mathrm{meV}$. The bias independent emission rate is $e_{n 0}=5.0 \cdot 10^{7} \mathrm{~s}^{-1}$. All other parameters are as in Fig.2(c), except for the $\mathrm{Ta}_{2} \mathrm{O}_{5}$ thicknesses that is set to $t_{D}=5 \mathrm{~nm}$. Area $\mathrm{A}=2500 \mathrm{~nm}^{2}$ and $n_{D}=100$. b. Charge versus ferroelectric field of the $\mathrm{Hf}_{0.5} \mathrm{Zr}_{0.5} \mathrm{O}_{2}-\mathrm{Ta}_{2} \mathrm{O}_{5}$ MFIM system for two different oxide thicknesses, $t_{D}$, and different trap densities $N_{T}$.

\section{S7 Numerical methods}

Numerical integration of Landau-Ginzburg-Devonshire dynamic equations. In this work the integration of the LGD equations is obtained with a specifically tailored implicit integrator with an adaptive error control. 
The implicit method has been chosen for two main reasons: a) the inherent numerical stability that allows us to use larger time steps compared to an explicit method; b) the robustness and effectiveness for the solution of numerically stiff problems having largely different time constants. ${ }^{3}$ The problem at study is in fact numerically stiff for simulations including interface traps, whose time constants vary in a large range depending on the trap energy and applied bias, and can be very different compared to the ferroelectric time constants. ${ }^{4}$

More specifically, we employed a second-order algorithm, namely the trapezoidal numerical integration method (also known as Crank-Nicolson method), that solves a differential equation $\frac{d x}{d t}=f(t, x)$ in time domain by using $x_{n+1}=x_{n}+\frac{h}{2}\left[f\left(t_{n}, x_{n}\right)+f\left(t_{n+1}, x_{n+1}\right)\right],{ }^{5}$ where $f$ is a generic function of time and of a state variable $x$, and $h=t_{n+1}-t_{n}$ is the time step. Because the above expression for $x_{n+1}$ involves variables evaluated at the instant $t_{n+1}$ both at the right- and at the left-hand side, at each integration step it is also required to solve a non-linear system of equations with a Newton-Raphson method, which involves itself the computation of the Jacobian matrix.

The error control, based on the number of the iterations of the Newton-Raphson algorithm, efficiently and automatically adapts the time-step $h$ to achieve a given accuracy for the results, thus allowing very fast simulations and overcoming the computational burden introduced by the resolution of the non-linear system.

Three-dimensional electrostatics simulations. For the MFIM capacitor sketched in Fig.1(a) the polarisation in each domain and the external voltage $V_{T}$ are the electric field sources of a three-dimensional (3D) electrostatic problem. In order to tackle such a 3D problem and eventually calculate the capacitances defined in Eq.S4, we used an on purpose developed simulation tool.

More precisely, we solve the following electrostatic problem in a connected region $\Omega$ of the 3-D Euclidean space

$$
\begin{cases}\operatorname{curl} \bar{E} & =\overline{0} \\ \operatorname{div} \bar{D} & =\rho \\ \bar{D} & =\varepsilon \bar{E},\end{cases}
$$

where $\varepsilon$ is the electric permittivity, $\bar{E}$ and $\bar{D}$ are the electric field and the electric displacement vectors, respectively. The material parameter $\varepsilon$ is assumed to be a positive scalar value which is piecewise uniform in each material region. The region boundary $\partial \Omega$ is partitioned into a set of $N^{\mathrm{c}}+1$ disjoint equipotential surfaces (electrodes) of perfect metals $\partial \Omega_{k}^{\mathrm{c}}$ and a set of $N^{\mathrm{i}}$ surfaces where the normal component of the electric field vanishes:

$$
\partial \Omega=\sum_{k=0}^{N^{\mathrm{c}}} \partial \Omega_{k}^{\mathrm{c}}+\sum_{k=1}^{N^{\mathrm{i}}} \partial \Omega_{k}^{\mathrm{i}} .
$$

Electrode $\partial \Omega_{0}^{\mathrm{c}}$ is considered as reference for all the voltages of the remaining electrodes, that are supposed to be assigned. $\bar{D} \cdot \bar{n}=0$ is set as boundary conditions on each $\partial \Omega_{k}^{\mathrm{i}}$, where $\bar{n}$ is the outwards oriented normal unit vector of $\partial \Omega$.

The solver implements an electrostatic formulation based on the electric scalar potential, ${ }^{6}$ that expresses physical laws directly in an algebraic form by using tools from algebraic topology. Physical variables are defined as fluxes or circulations on oriented geometric elements of a pair of dual interlocked computational grids, while physical conservation laws are enforced strongly in a metric-free fashion by means of incidence matrices between grid elements. The metric and the material information are encoded in the discrete counterpart of the constitutive laws of materials, also referred to as material matrices. The stability and consistency of the method are guaranteed by precise properties (symmetry, positive definiteness, geometric consistency) that material matrices have to fulfill.

The main advantage of this approach with respect to the conventional Finite Element Method is that the material matrices for arbitrary polyhedral elements can be geometrically defined, by simple closed-form expressions, in terms of the geometric elements of the primal and dual grids. 


\section{References}

${ }^{1}$ M. Hoffmann, M. Pešić, S. Slesazeck, U. Schroeder, and T. Mikolajick, "On the stabilisation of ferroelectric negative capacitance in nanoscale devices," Nanoscale, vol. 10, pp. 10891-10899, 2018. doi: 10.1039/C8NR02752H.

${ }^{2}$ A I. Khan, K. C. andJ. P. Duarte, Z. Lu, A. Sachid, S. Khandelwal, R. Ramesh, C. Hu and S. Salahuddin, "Negative Capacitance in Short-Channel FinFETs Externally Connected to an Epitaxial Ferroelectric Capacitor," IEEE Electron Device Letters, vol. 37, no. 1, pp. 111-114, 2016.

${ }^{3}$ G. Dahlquist and B. Lindberg, "On some implicit one-step methods for stiff differential equations," Tech. Report, Department of Information Processing, Royal Institute of Technology, Stockholm, 1973.

${ }^{4}$ T. Rollo and L. Daniel and D. Esseni, "Accurate and Efficient Dynamic Simulations of Ferroelectric Based Electron Devices," 2019 International Conference on Simulation of Semiconductor Processes and Devices (SISPAD),pp. 1-4, 2019. doi: 10.1109/SISPAD.2019.8870373.

${ }^{5}$ G. Dahlquist, “A Special Stability Problem for Linear Multistep Methods," BIT Numerical Mathematics, vol. 3, pp. 27-43, 1963. doi: 10.1007/BF01963532.

${ }^{6}$ R. Specogna, "Complementary geometric formulations for electrostatics," International Journal for Numerical Methods in Engineering, vol. 86, pp. 1041-1068, 2011. 